\title{
Rotation and accretion of very low mass objects in the $\sigma$ Ori cluster ${ }^{\star}$
}

\begin{abstract}
A. Scholz ${ }^{\star \star}$ and J. Eislöffel ${ }^{\star \star}$
Thüringer Landessternwarte Tautenburg, Sternwarte 5, 07778 Tautenburg, Germany

Received 27 June 2003 / Accepted 27 December 2003

Abstract. We report on two photometric monitoring campaigns of Very Low Mass (VLM) objects in the young open cluster around $\sigma$ Orionis. Our targets were pre-selected with multi-filter photometry in a field of 0.36 sqdeg. For 23 of these objects, spanning a mass range from 0.03 to $0.7 M_{\odot}$, we detect periodic variability. Of these, 16 exhibit low-level variability, with amplitudes of less than $0.2 \mathrm{mag}$ in the $I$-band, which is mostly well-approximated by a sine wave. These periodicities are probably caused by photospheric spots co-rotating with the objects. In contrast, the remaining variable targets show highlevel variability with amplitudes ranging from 0.25 to $1.1 \mathrm{mag}$, consisting of a periodic light variation onto which short-term fluctuations are superimposed. This variability pattern is very similar to the photometric behaviour of solar-mass, classical $\mathrm{T}$ Tauri stars. Low-resolution spectra of a few of these objects reveal strong $\mathrm{H} \alpha$ and Ca-triplet emission, indicative of ongoing accretion processes. This suggests that $5-7 \%$ of our targets still possess a circumstellar disk. In combination with previous results for younger objects, this translates into a disk lifetime of 3-4 Myr, significantly shorter than for solar mass stars. The highly variable objects rotate on average slower than the low-amplitude variables, which is expected in terms of a disk-locking scenario. There is a trend towards faster rotation with decreasing mass, which might be caused by shortening of the disk lifetimes or attenuation of magnetic fields.
\end{abstract}

Key words. techniques: photometric - stars: low-mass, brown dwarfs - stars: rotation - stars: formation - stars: activity stars: magnetic fields

\section{Introduction}

Open clusters are ideal environments to study stellar properties and evolution, because they contain a homogeneous population of objects with known distance, metallicity, and age. Recent deep surveys have unveiled the population of several open clusters far down into the substellar regime. Examples are the surveys by Moraux et al. (2003), Pinfield et al. (2000) and Zapatero Osorio et al. $(1999,1997)$ in the Pleiades, and the work of Barrado y Navascués et al. in IC 2391 (2001) and $\alpha$ Per (2002). In two cases, namely in the clusters around $\sigma$ Ori (Zapatero Osorio et al. 2000) and the Orion Trapezium Cluster (Lucas \& Roche 2000), even isolated planetary mass objects were found. Thanks to these surveys, large samples of Very Low Mass (VLM) objects are known today, among which we conveniently will subsume all objects with masses below

Send offprint requests to: A. Scholz, e-mail: scholz@tls-tautenburg.de

* Table 7 is only available in electronic form at the CDS via anonymous ftp to cdsarc.u-strasbg.fr $(130.79 .128 .5)$ or via http: //cdsweb.u-strasbg. fr/cgi-bin/qcat?]/A+A/419/249

$\star \star$ Visiting Astronomer at the German-Spanish Astronomical Centre, Calar Alto, operated by the Max-Planck-Institute for Astronomy, Heidelberg, jointly with the Spanish National Commission for Astronomy.
$0.4 M_{\odot}$, including very low mass stars, brown dwarfs and freefloating planetary mass objects. All objects with masses below this $0.4 M_{\odot}$ limit are thought to be fully convective (Chabrier \& Baraffe 2000), giving a physical motivation for this definition of VLM objects.

Wide-field photometric monitoring is a powerful tool to investigate properties of large object samples: if an object exhibits asymmetrically distributed surface features, e.g. magnetically induced star spots, its flux will be modulated with the rotation period. Hence, period search in the lightcurve allows the determination of the (projection-free) rotation period. The amplitude of the periodicity, in turn, depends on the properties of the star spots, allowing conclusions about surface activity processes. Pointing to dense open cluster fields, such monitoring campaigns become very efficient, as one can register many objects contemporaneously within one field of view.

Compared to solar-mass stars, there is a significant lack of known rotation periods for VLM objects. Photometric monitoring studies delivered a small number of periods for evolved VLM stars and Brown Dwarfs (Bailer-Jones \& Mundt 1999, 2001; Tinney \& Tolley 1999; Martín et al. 2001; Clarke et al. 2002; Gelino et al. 2002). Only three periods are known for VLM objects with ages between 50 and $100 \mathrm{Myr}$ (Martín \& Zapatero Osorio 1997; Terndrup et al. 1999). Two periods for VLM members of $\sigma$ Ori were published by 
Bailer-Jones \& Mundt (2001). Recently, Joergens et al. (2003) report on 5 measured periods for Brown Dwarfs and VLM stars in the very young ChaI star forming region. Our own study in the young open cluster IC 4665 (age $36 \mathrm{Myr}$ ) delivered several rotation periods for VLM objects (Eislöffel \& Scholz 2002).

Observations of very young VLM objects are of special interest, because they can deliver clues about their formation process. Recent results suggest that objects in the substellar regime form similar to stars. Several authors detected typical T Tauri star phenomena on VLM objects, e.g. outflow processes (Fernández \& Comerón 2001) and mid-infrared excesses attributed to the presence of a circumstellar disk (Natta \& Testi 2001; Apai et al. 2002; Jayawardhana et al. 2003). Moreover, López Martín et al. (2004) and Liu et al. (2003) demonstrate a correlation between $\mathrm{H} \alpha$ emission and mid-infrared excess for Brown Dwarfs down to $0.02 M_{\odot}$, which they interpret as an indication for ongoing accretion.

Solar-mass T Tauri stars show various types of photometric variability. Active accretion processes often manifest themselves by large amplitude variations showing several possible periods (e.g., Fernández \& Eiroa 1996; Bouvier et al. 1995; Herbst et al. 2000). If VLM objects undergo a T Tauri phase as well, they should exhibit a similar photometric behaviour. Hence, photometric monitoring can deliver an independent contribution to the ongoing debate about VLM object formation.

The $\sigma$ Ori cluster is an appropriate target for such a study, because it is relatively nearby ( $350 \mathrm{pc}$, Béjar et al. 1999), has negligible extinction $\left(E_{B-V}=0.05\right.$, Béjar et al. 1999) and an age of $3 \mathrm{Myr}$ (Zapatero Osorio et al. 2002). The extended work by Béjar et al. and Zapatero Osorio et al. revealed a rich VLM population. We monitored this cluster in two photometric time series. Complementary observations, presented in Sect. 2 , identified the cluster members in the time series field. We report on the monitoring campaigns in Sect. 3 and the time series analysis in Sect. 4. The following sections describe the results of out lightcurve analysis: we first establish two origins for the observed variability (Sect. 5). In Sect. 6, we then concentrate on the investigation of the rotation periods. Section 7 contains the discussion of low-resolution spectra for highly variable objects. Finally, in Sect. 8, we present our conclusions.

\section{Selection of VLM objects in the $\sigma$ Ori cluster}

We searched for VLM members of the $\sigma$ Ori cluster using the 2-m Schmidt camera at the Thüringer Landessternwarte Tautenburg (TLS) and the $1.23-\mathrm{m}$ telescope on Calar Alto (CA) in combination with infrared photometry from 2 MASS $^{1}$. Figure 1 shows the $36^{\prime} \times 36^{\prime}$ TLS field and the $17^{\prime} \times 17^{\prime} \mathrm{CA}$ field in the north of $\sigma$ Ori. These survey images were obtained as part of the time series campaigns (see next section). The CA field was observed in the $R$ - and $I$-band with exposure times of 30 and $600 \mathrm{~s}$, respectively. For the TLS field, we used a 600 -s-exposure in the $I$-band. These images were reduced with the standard routines within the $c c d r e d$ package

\footnotetext{
${ }^{1}$ Catalogue available under http://www.ipac.caltech.edu/2mass
}

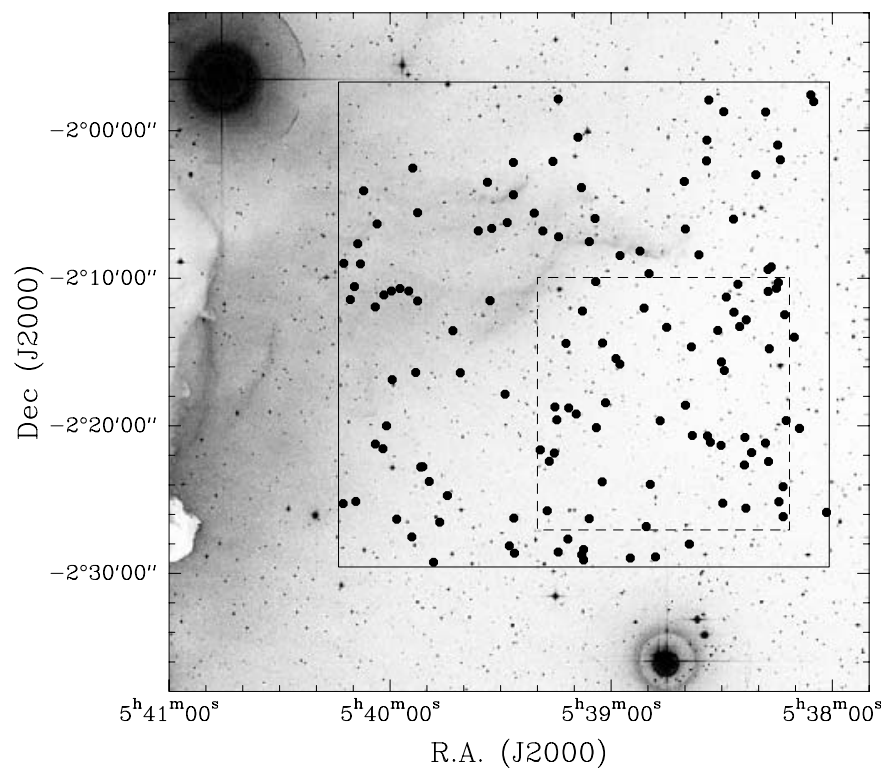

Fig. 1. Time series fields in the $\sigma$ Ori cluster: the TLS field is indicated with solid, the CA field with dashed lines. The positions of the cluster member candidates (see Table 7) are overplotted. $\sigma$ Ori is the bright star near the bottom of the field.

of $\mathrm{IRAF}^{2}$, including overscan and bias subtraction, as well as flatfield correction. The $I$-band fringe pattern caused by night sky emission was corrected with a fringe mask constructed from blank field images. The sigma-clipped median of these frames ("superflat") was smoothed and subtracted from the original superflat, delivering the required fringe mask. Because the fringe mask contains only an average fringe pattern, this mask must be scaled to properly subtract the fringes in the individual images. The scaling factor was determined as the ratio of the fringe amplitude in the fringe mask and in the respective image.

The pixel positions of the objects in the $I$-band images were determined with the SExtractor software (Bertin \& Arnouts 1996). The respective positions in the $R$-band image were found by a linear transformation determined with several bright stars whose pixel positions were manually determined in $R$ and $I$. The pixel positions were transformed to sky coordinates with the known sky coordinates of unsaturated HST guide stars (Morrison et al. 2001) in our field using the ccmap routine of IRAF. The coordinate precision is $\pm 11^{\prime \prime} 0$.

Instrumental magnitudes for all objects in the TLS and CA catalogues were determined by PSF fitting (TLS) or aperture photometry (CA) using the daophot package within IRAF (Stetson 1987). The PSF fitting photometry was considered to be more appropriate for the TLS data, because the poor seeing turned the cluster into a "crowded field". Near-infrared photometry in $J, H$ and $K$ for both fields was obtained from the 2MASS database.

${ }^{2}$ IRAF is distributed by National Optical Astronomy Observatories, which is operated by the Association of Universities for Research in Astronomy, Inc., under contract with the National Science Foundation. 
In order to calibrate the CA images, we observed two standard fields (Landolt 1992) at an airmass comparable with that of the $\sigma$ Ori field. $I$-band zero-point and colour coefficient were derived fitting the instrumental magnitudes and the catalogue magnitudes with the following relation ( $I$ - Landolt magnitude; $i, r$ - instrumental magnitudes):

$I=i_{\mathrm{CA}}+Z P+C\left(r_{\mathrm{CA}}-i_{\mathrm{CA}}\right)$.

With the resulting parameters $(Z P=3.45, C=0.171)$, the $\sigma$ Ori magnitudes were converted to the Landolt system. The high colour coefficient points at significant differences between the CA and the Landolt $I$-band. Since the Landolt standards are mainly stars with $R-I<1.0$, one has to be cautious to apply this colour correction to very red targets. For such objects, we may overestimate the $I$-band flux.

The CA field completely overlaps with the TLS field. Therefore, the TLS magnitudes were calibrated using objects contained in both catalogues. We noted a significant colour dependency of the zero-point offset between CA and TLS $I$-band, therefore we applied a colour correction using the 2MASS $J$-band magnitudes. The following transformation was used to convert TLS magnitudes into the Landolt system.

$I=i_{\mathrm{TLS}}+Z P+C\left(i_{\mathrm{TLS}}-J\right)$.

After this procedure, $I$-band magnitudes from both telescopes are available in the same photometric system.

Cluster member candidates were selected from $(I, I-$ $J$ ) colour magnitude diagrams (Fig. 2). In both diagrams, a cumulation of objects to the right of the field stars is clearly visible. To determine the position of this cumulation precisely, we divided the colour magnitude diagrams (CMD) in horizontal bins of $\Delta I=1 \mathrm{mag}$. The histograms of the $I-J$ colour of these bins typically show a broad maximum on the left and a second smaller maximum on the right side. This second peak indicates the position of the cluster isochrone. We registered the $I-J$ value of this peak for each bin in both diagrams and fitted these values linearly. This fit, the empirical isochrone, shifted $0.3 \mathrm{mag}$ to the left to account for photometric errors and the uncertainty of the derived isochrone, was used as dividing line between cluster member candidates and field stars (see Fig. 2, straight line). All objects to the right side of this separation line are potential low-mass cluster members. Some of these objects clearly appear reddened in the CMD. Since extinction towards $\sigma$ Ori is negligible (Béjar et al. 1999), this is a sign for intrinsic reddening, as found for objects with similar masses in the same cluster (Oliveira et al. 2002). We selected 55 candidates from the CA and 112 from the TLS diagram. The TLS candidates contain 28 objects also identified with the CA photometry. The remaining CA candidates are either too bright for the TLS photometry or not resolved in the TLS image.

In Fig. 2, we also indicate the position of the Baraffe et al. (1998) isochrone for the $\sigma$ Ori cluster. The $I-J$ colours of our candidates are significantly smaller than those from the evolutionary track for the appropriate age. This difference is possibly caused by insufficient colour correction in the $I$-band (see above) as well as shortcomings in the modeled BD atmospheres
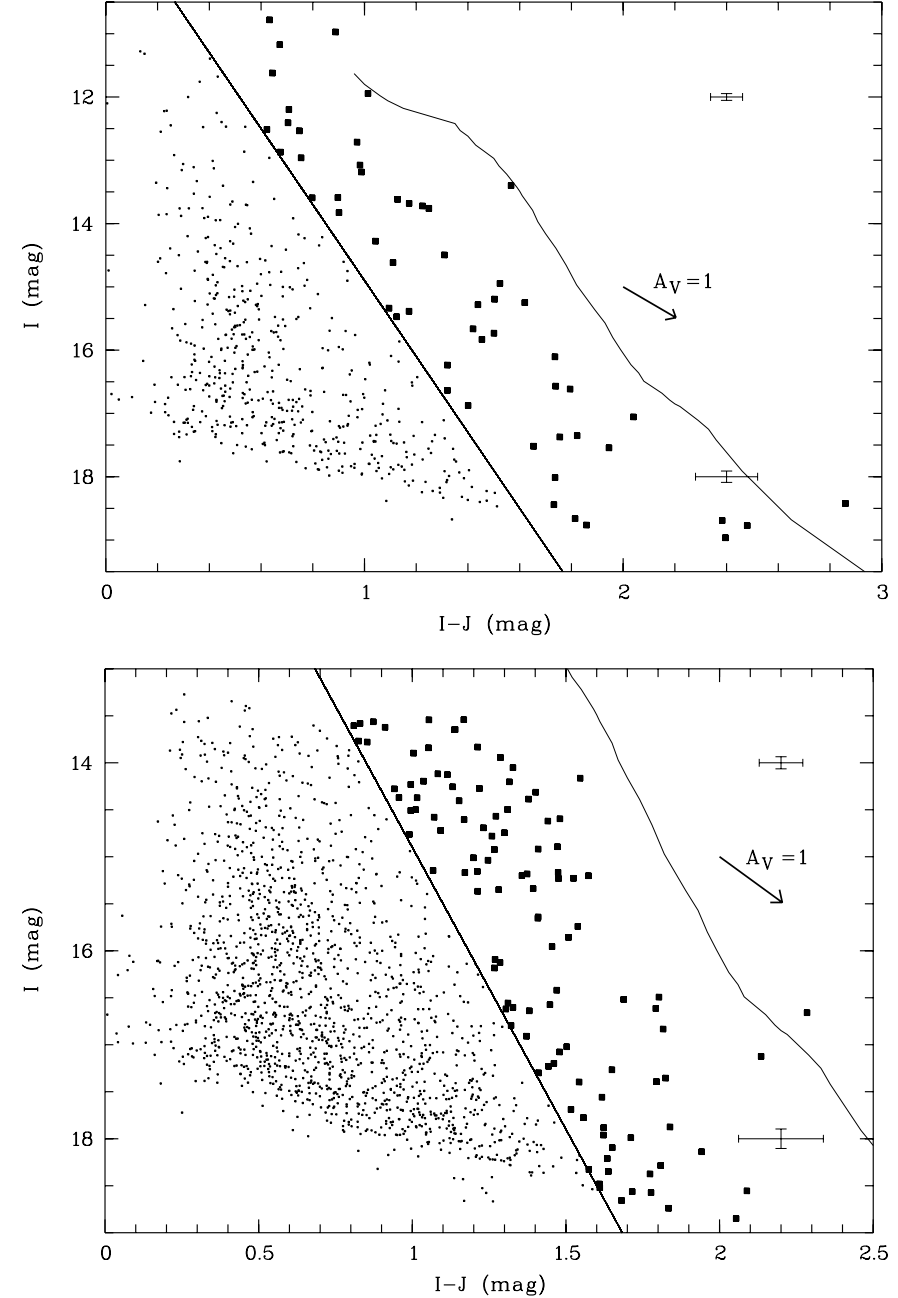

Fig. 2. $(I, I-J)$ colour magnitude diagram for the CA (upper panel) and the TLS field (lower panel). All probable cluster members are marked as larger dots. Error bars indicate typical photometry errors for the candidates. The separation line between cluster member candidates and field objects is shown as a straight line. The position of the $3 \mathrm{Myr}$ isochrone from Baraffe et al. (1998) is indicated by the righthand line. The arrow shows the reddening vector for $A_{\mathrm{V}}=1 \mathrm{mag}$.

and colours. However, it is valid to compare the 2MASS photometry with the Baraffe et al. isochrone. Systematic mismatches between the 2MASS photometric system and the CIT system used for the model isochrones are safely below $3 \%$ (Carpenter 2001) and thus negligible for our purposes.

We used the $R$-band photometry from CA to verify the status of the CA candidates. With one exception (which was rejected), all CA candidates with detection in the $R$-band image are situated on the red side of the $(I, R-I) \mathrm{CMD}$. As a further test, we compared the $(J-H)$ colours of our candidates with the models of Baraffe et al. (1998). The $(J-H)$ values were preferred over the $(J-K)$ colour because the latter might be influenced by excess radiation from a circumstellar disk, as already found for VLM objects of similar age (Muench et al. 2001; Oliveira et al. 2002). Figure 3 shows the $(J, J-H)$ CMD for all our candidates and the Baraffe et al. isochrones for the $\sigma$ Ori cluster. Our VLM candidates fall around the $3 \mathrm{Myr}$ isochrone (solid line). The $1 \mathrm{Myr}$ (dotted line) and $5 \mathrm{Myr}$ (dashed line) isochrones are indistinguishable from 


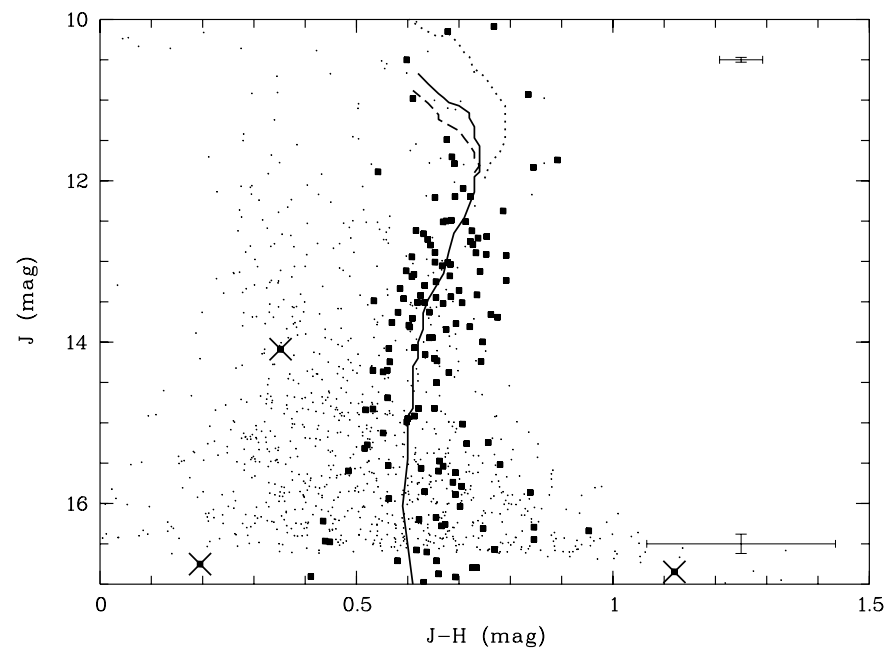

Fig. 3. $(J, J-H)$ colour magnitude diagram for all candidates (plotted as filled squares). Error bars indicate typical photometry errors for the candidates. The $3 \mathrm{Myr}$ isochrone for $\sigma$ Ori (Baraffe et al. 1998) is shown as a solid line. For bright objects, the isochrones for $1 \mathrm{Myr}$ (dotted line) and $5 \mathrm{Myr}$ (dashed line) differ significantly from the $3 \mathrm{Myr}$ track and are therefore also shown. Objects whose $J-H$ colour deviates clearly from the cluster isochrone are marked with crosses and excluded. The positions of 1000 arbitrarily selected field stars are shown as small dots.

the 3 Myr track for $J>12$ mag, but show clear deviations for brighter objects. We excluded one CA and two TLS candidates which are clear outliers and thus probably non-members of the cluster. It should be noted that the $(J, J-H) \mathrm{CMD}$ is not particularly well suited to discriminate between cluster members and field stars, as seen in Fig. 3 from the 2MASS data for arbitrarily selected objects in the region around $\sigma$ Ori. In contrast to the $(I, I-J) \mathrm{CMD}$, there is no clear separation of field stars and cluster members. Therefore, the $(J, J-H)$ CMD will only reveal obvious false detections and does not signficantly reduce the contamination rate of our sample (see below).

The remaining candidate list comprises $52 \mathrm{CA}$ and 110 TLS objects, including 27 double detections. Coordinates and photometry for these 135 objects are listed in Table 7. Masses for these candidates were estimated by fitting the $3 \mathrm{Myr}$ isochrone of Baraffe et al. for the $J$ - and $H$-band with a low degree polynomial and applying the fit to the $J$ - and $H$-magnitudes. This gives us two mass estimates, one based on $J$-, the other on $H$-band photometry. We found both values to be very similar, and use their average as final mass (see Table 7). The theoretical isochrone covers the mass range from 0.02 to $1.4 M_{\odot}$. The mass estimate for fainter or brighter objects is thus not possible with these models. For these (very few) targets, we only give an upper or lower limit for the mass.

Our candidate list may be contaminated by fore- and background objects. However, the spectroscopic follow-up observations of Béjar et al. (2001) for an object sample selected with similar photometric criteria suggest that contamination rates are low. A 7 arcmin high stripe in the southern part of our field is also covered by the survey of Béjar et al., although they are only sensitive to objects with $I>15$. In the overlapping region, we found 18 cluster member candidates in this magnitude range. Of these candidates, 11 (61\%) are also identified by
Table 1. Cluster member candidates which were previously identified by Béjar et al. (2001) as possible $\sigma$ Ori members. We also list the $I$-band magnitudes given by Béjar et al. (2001) as well as the difference to our $I$-band photometry.

\begin{tabular}{llcc}
\hline \hline No. & Name & $I_{\mathrm{C}}(\mathrm{mag})$ & $\Delta I$ \\
\hline 4 & SOri J053813.1-022410 (SOri 13) & 16.41 & 0.68 \\
9 & SOri J053817.1-022228 (SOri 9) & 15.81 & 0.56 \\
22 & SOri J053829.5-022517 (SOri 29) & 17.23 & 0.66 \\
33 & SOri J053849.2-022358 & 16.81 & 0.98 \\
49 & SOri J053915.1-022152 (SOri 38) & 17.64 & 0.10 \\
73 & SOri J053848.0-022854 (SOri 15) & 16.79 & 0.83 \\
81 & SOri J053907.4-022908 (SOri 20) & 17.32 & 0.90 \\
83 & SOri J053907.9-022848 (SOri 8) & 15.74 & 0.37 \\
86 & SOri J053911.7-022741 (SOri 1) & 15.08 & 0.36 \\
106 & SOri J053944.4-022445 (SOri 10) & 16.08 & 0.34 \\
108 & SOri J053948.1-022914 & 18.92 & 0.82 \\
\hline
\end{tabular}

Béjar et al. (2001). Assuming the completeness of their survey, this gives a first rough estimate of up to $40 \%$ for the contamination rate. Table 1 contains our identification numbers as well as object names and $I$-band photometry of Béjar et al. (2001) for common candidates. Our I-band magnitudes for these objects are systematically smaller than those given in Béjar et al. (2001). The offset between both photometric systems (given in the last column of Table 1 ) increases with the $I-J$ colour, which may result from insufficient colour correction in the absolute photometry. Since the objects in Table 1 cover only a small range in $I-J$ (8 of 11 objects have $0.4<I-J<0.7)$, it is not possible to derive a colour correction that would bring all objects onto the same system.

An alternative estimate for the number of contaminating field stars can be obtained with the Besancon Galaxy model ${ }^{3}$ (Robin \& Crézé 1986). The simulation delivers star counts depending on Galactic coordinates, colour and brightness. A simulated $(I, R-I) \mathrm{CMD}$ for a $1 \mathrm{sq}$ field centred on $\sigma$ Ori contains 90 objects in a 0.4 mag wide stripe around the cluster isochrone of Baraffe et al. (1998). Scaling to the appropriate field sizes, we calculate that 7 of the 52 CA candidates (13\%) and 32 of the 110 TLS (29\%) candidates are field stars, i.e. a significantly lower rate than in our first estimate. Since interstellar extinction variations might hamper the CMD simulation, we assume conservatively that about $30 \%$ of our candidates may not be members of the $\sigma$ Ori cluster. However, the definite decision about the cluster membership of our candidates must be postponed until we have obtained follow-up spectroscopy for all of them. Our preliminary cluster member list in Table 7 will be used in the following as target list for the variability study.

\section{Time series observations}

\subsection{Observing campaigns and data reduction}

The region with our cluster member candidates was monitored in two I-band time series campaigns, with the TLS Schmidt

\footnotetext{
3 These simulations are available online under http://www.obs-besancon. fr/www/modele/modele_ang.html
} 
Table 2. Monitoring campaigns.

\begin{tabular}{|c|c|c|}
\hline & TLS Schmidt & CA1.23 \\
\hline Aperture & $1.34 \mathrm{~m}$ & $1.23 \mathrm{~m}$ \\
\hline CCD size & $2 \mathrm{k} \times 2 \mathrm{k}$ & $2 \mathrm{k} \times 2 \mathrm{k}$ \\
\hline Pixel scale & 1".26/pix & $0.5 /$ pix \\
\hline Observing nights & 16-26 Jan. 2001 & 16-27 Dec. 2001 \\
\hline Mean seeing & $2 \prime .5$ & $1{ }^{\prime \prime} 8$ \\
\hline CCD images & 77 & $78 / 78^{*}$ \\
\hline
\end{tabular}

* The CA time series consists of 78 short and 78 long exposures, which were taken alternately.

telescope and with the 1.23-m telescope on Calar Alto (CA). Details of these runs are given in Table 2. During the CA campaign, we took alternating short (30s) and long (600s) exposures to extend the dynamical range of the time series photometry. In both campaigns, we pointed to the same position in the sky to within $\approx 10^{\prime \prime}$ in all monitoring nights.

Both observing runs span at least ten days. The distribution of the datapoints over the observing run, however, is irregular because of variable weather conditions. The number of datapoints per night varies from 0 to 27 . Figure 4 shows the datapoint distribution for both runs.

Image reduction and photometry of both campaigns was done following the recipes explained in the previous section. An object catalogue was created for the deepest time series image of both campaigns. For each time series image, we determined the spatial offset between the object catalogue and the positions in the actual image. Applying this offset to the object catalogue and centering these new positions on the respective image generates an object catalogue for each image. We used a fixed sample of PSF stars for each night of the TLS time series. After the photometry procedure, we obtained lightcurves for all objects in the respective object catalogue.

\subsection{Relative calibration}

The lightcurves of both campaigns were calibrated using an average lightcurve from a set of non-variable reference stars in the observed field. This is necessary to correct for changing atmospheric conditions as well as the variable airmass over the time series. In the following, we describe the selection process for the reference stars.

First, a sample of stars was selected, whose photometry errors were below $0.1 \mathrm{mag}$ in all images. To reject variable objects from this initial reference star list, we examined the variability of each star with respect to the average lightcurve of all other stars. We used the following procedure based on the routine by Allain (1994). The number of a reference star is indicated with $i=1 \ldots N_{R}$, the number of an image with $j=1 \ldots N_{B}$.

1. Averaging the instrumental magnitudes for each star

$$
\overline{m_{i}}=\frac{1}{N_{B}} \sum_{j=1}^{N_{B}} m_{i}\left(t_{j}\right)
$$
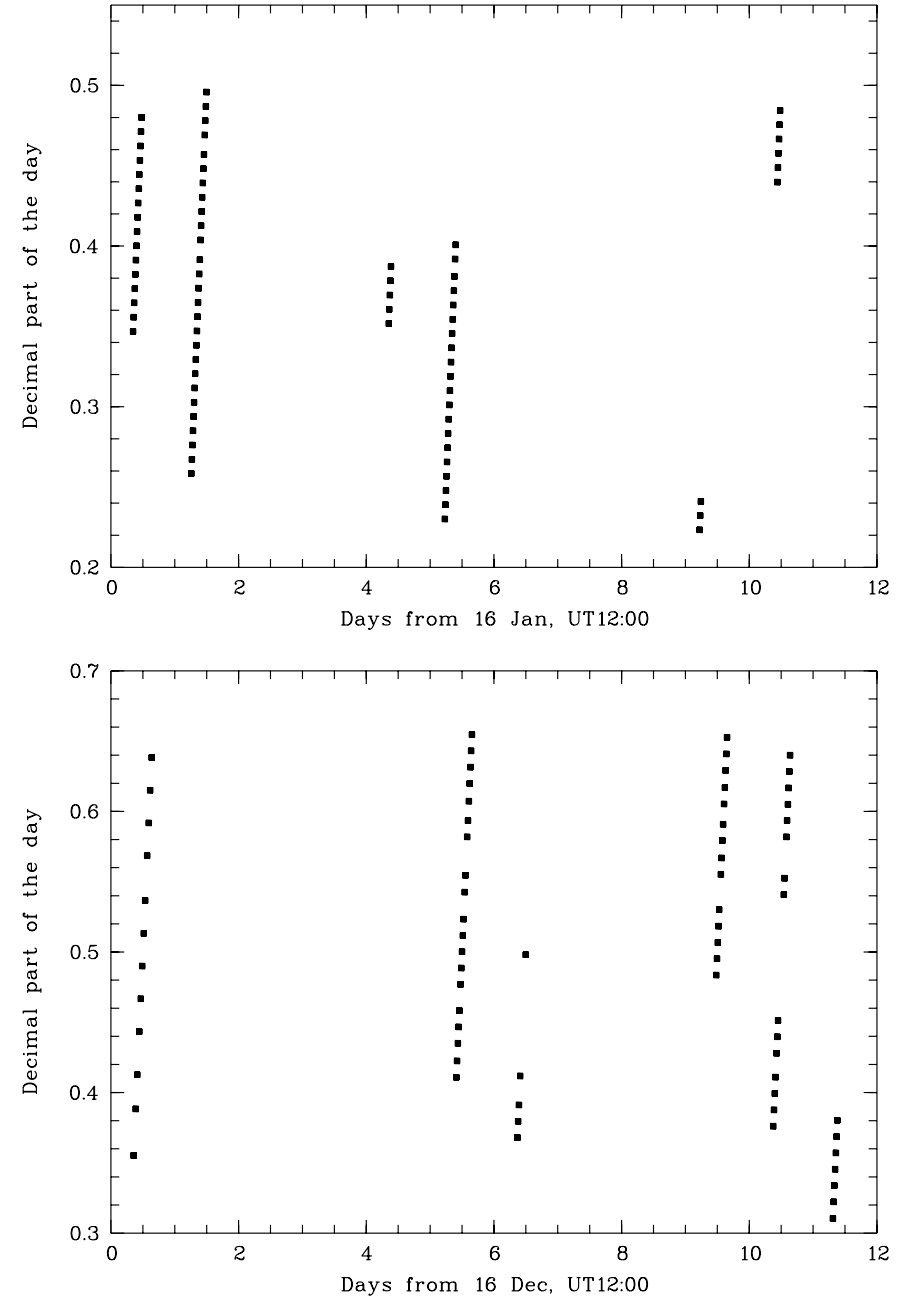

Fig. 4. Datapoint distribution for the TLS (upper panel) and the CA run (lower panel). Plotted is the non-integer fraction of the observing times against the observing times.

2. subtraction of this average from every value of the time series for every star

$m_{i}^{0}\left(t_{j}\right)=m_{i}\left(t_{j}\right)-\overline{m_{i}}$

3. calculation of average $\overline{m_{j}^{0}}$ and standard deviation $\sigma_{j}$ for the differences from Eq. (4)

$\overline{m_{j}^{0}}=\frac{1}{N_{R}} \sum_{i=1}^{N_{R}} m_{i}^{0}\left(t_{j}\right)$

$\sigma_{j}=\sqrt{\frac{1}{N_{R}-1} \sum_{i=1}^{N_{R}}\left(m_{i}^{0}\left(t_{j}\right)-\overline{m_{j}^{0}}\right)^{2}} ;$

4. rejection of obvious "bad" images by examination of $\sigma_{j}$ ( $N_{B}^{\prime}$ is the number of the remaining images);

5. repeated execution of steps 1-3 without the rejected images;

6. calculation of a quality number test $t_{i}$, which allows for every star an examination of its intrinsic variability compared to 
all other reference stars; if $\left|m_{i}^{0}\left(t_{j}\right)-\overline{m_{j}^{0}}\right| \geq \sigma_{j}$ is test $_{i, j}=1$, elsewhere 0

$$
\text { test }_{i}=\sum_{j=1}^{N_{B}^{\prime}} \text { test }_{i, j}
$$

After the described procedure, the stars with test $t_{i}<3$ were selected as reference stars. We obtained $\approx 170$ reference stars for the TLS and $\approx 80$ for the CA campaign. For all images, the average brightness of the reference stars was determined.

$\overline{m^{\mathrm{ref}}}\left(t_{j}\right)=\frac{1}{N_{\text {ref }}} \sum_{i=1}^{N_{\text {ref }}} m_{i}^{\mathrm{ref}}\left(t_{j}\right)$.

This mean lightcurve was subtracted from all time series. Thus, we obtained extinction corrected relative magnitudes $m^{\text {rel }}\left(t_{j}\right)$ from the instrumental magnitudes for all objects. $m\left(t_{j}\right)$.

$m^{\mathrm{rel}}\left(t_{j}\right)=m\left(t_{j}\right)-\overline{m^{\mathrm{ref}}}\left(t_{j}\right)$

\subsection{Optimal image subtraction}

For CCD images from the 1.23-m telescope on the Calar Alto, a dedicated image analysis package has been developed by the Wendelstein Calar Alto Pixellensing Project (WeCAPP, Riffeser et al. 2001) team. This software is based on "Optimal Image Subtraction" (OIS, Alard \& Lupton 1998). After astrometric and photometric alignment, it performs an image convolution with respect to a reference frame (Gössl \& Riffeser 2002). The reference image is obtained by coaddition of the time series images with the best seeing. For the long exposure time series from CA, we used this pipeline to check the quality of our lightcurves from our already described "classical" photometry. Because of bad image quality, six images were not used for this procedure.

The basic idea of difference image analysis algorithms like OIS is to measure only the variability and not the constant portion of the star's flux. The result of the pipeline are frames which contain only variable sources. On these frames, we performed aperture photometry for the previously determined object catalogue. The resulting fluxes were transformed to relative fluxes by division by the flux of the respective object in the reference image. Relative fluxes were transformed to relative magnitudes with $m_{\text {rel }}=-2.5 \log _{10}\left(1+f_{\text {rel }}\right)$.

To compare the results from OIS with those from daophot, we determined the photometric precision of both measurements: after excluding $3 \sigma$ outliers, we calculated average and rms for all lightcurves. The lightcurves of the brightest objects scatter with $4 \mathrm{mmag}$ in the OIS data, $3 \mathrm{mmag}$ less than with classical photometry. Throughout the usable magnitude range, OIS improves the precision by several mmag. Therefore, we used the lightcurves from the WeCAPP software for the further analysis. The short exposure CA images, however, cannot be reduced properly with the OIS pipeline, since they do not contain enough stars for the fitting process.

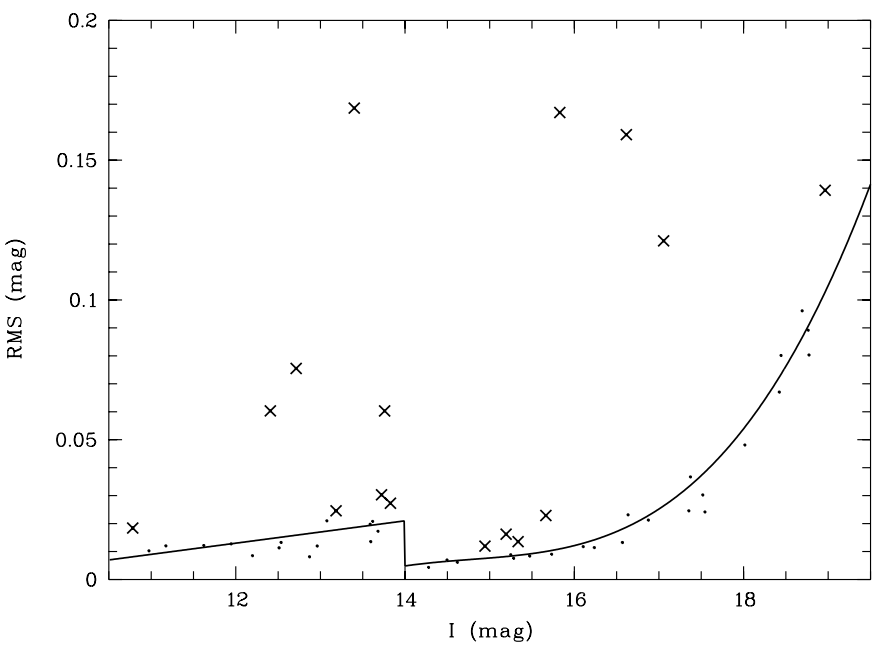

Fig. 5. Root mean square of the time series photometry vs. I-band magnitude for all CA candidates. The solid line is the mean rms of the relative photometry, determined by fitting the rms of all objects in the field. Below $I=14$ mag the long exposure photometry is influenced by saturation, brighter targets were analysed with the short exposure lightcurves. All objects marked with a cross are variable at the $99 \%$ level.

Three obstacles prevented a successful application of OIS to the TLS data:

- the pixel scale of the TLS Schmidt camera is roughly two times that of the CCD at the 1.23-m telescope on CA. This leads to an undersampled PSF, which makes exact image centering and image folding difficult;

- the sky background in the TLS images was in most cases $>25000$ ADU, because of snow cover around the observatory. Therefore, moderately crowded open cluster fields with many bright stars lead to many saturated pixels which are useless for difference image analysis and decrease the precision of the fitting process;

- every bright star causes a so-called Schmidt ghost in the image, disturbing the fitting process. However, if the ghosts were masked so that the fit can be done without them, we would loose a considerable fraction of the targets.

For all these reasons, we returned to "classical" PSF fitting photometry for the TLS images. High background caused by light reflection from snow, bad seeing and variable atmospheric conditions limited the precision of the TLS lightcurves. Here, we reach 0.015 mag for the brightest stars.

\section{Time series analysis}

\subsection{Generic variability test}

The candidate lightcurves of both campaigns were analysed with a unique procedure. In a first step, we examined all lightcurves visually to register obvious signs of rapid variability. In particular, we looked for sudden brightness eruptions (e.g. flares), eclipses or totally irregular variability. This search was not successful. It turned out that some TLS objects are either too bright or too faint to be detected in most of the images; time series analysis is not possible for these targets. 


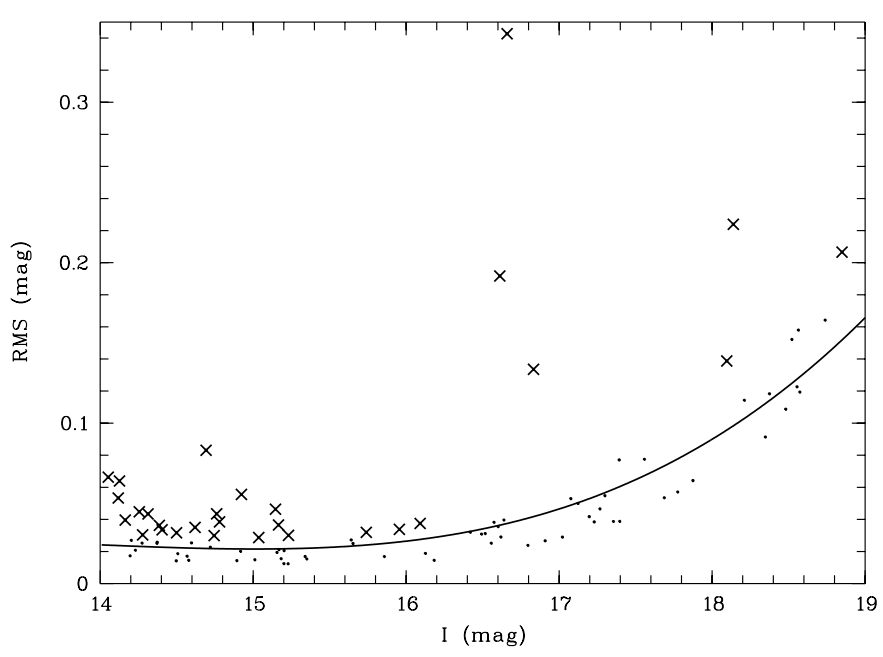

Fig. 6. Root mean square of the time series photometry vs. $I$-band magnitude for all TLS candidates. The solid line is the mean rms of the relative photometry, determined by fitting the rms of all objects in the field. All objects marked with a cross are variable at the $99 \%$ level. At the bright end, the variability test is contaminated by extinction residuals and beginning saturation.

Subsequently, the lightcurves were filtered: in three iterations, we excluded $3 \sigma$ outliers, but only if they were framed by other datapoints to avoid the exclusion of intrinsic short-term variability. The root mean squares of the filtered lightcurves are listed in Table 7 and plotted in Figs. 5 and 6. The solid lines in these figures indicate our photometric precision determined by fitting the rms of all lightcurves with low degree polynomials. This average rms was compared to the rms of the candidates with a statistical F-test, which is particularly well-suited for the comparison of scatter in data. All variable objects at a significance level of $99 \%$ are marked with a cross (and with a "v" in Table 7).

Of the 52 CA candidates, $16(30 \%)$ show significant variability. In contrast, all objects in our images, i.e. mostly field stars, have a much smaller fraction of variable objects $(\approx 10 \%)$. It is obvious from Fig. 5 that the fraction of variable objects as well as the variability amplitude does not change significantly over the entire magnitude range, which corresponds to masses down to $0.02 M_{\odot}$. Figure 6 shows the same data for the TLS run. Unfortunately, strongly variable conditions and high background lead to strongly scattered rms for bright objects. Additionally, the high image background prevents the detection of bright and highly variable targets, since they would be saturated around their lightcurve maxima. Therefore, the data for targets with $I<15$ mag are of limited use. However, the plot again shows no decrease of variability amplitude with I-magnitude (and hence mass). Interestingly, one variable substellar target (No. 108) was identified with an X-ray source by Mokler \& Stelzer (2002), confirming that this object is an active Brown Dwarf.

Summarizing, these are the two results from this test: a) the young VLM objects in our observed field contain a large fraction of variable objects; $b$ ) the fraction of variable objects and the variability amplitude is independent of mass from 0.5 down to $0.02 M_{\odot}$.

\subsection{Period search}

Our period search is based on the widely used Scargle periodogram (Scargle 1982). Compared to the classical periodogram, this modified version has one advantage: the probability that a periodogram peak at a given frequency has the height $z$ should be simply $P(z)=\exp (-z)$, even for nonuniformly sampled datasets. This makes the Scargle periodogram particularly well-suited for astronomical time series with their characteristic gaps between the observing nights.

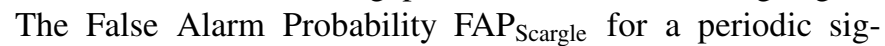
nal is the probability that a peak at any frequency has the height $z$ : $\operatorname{FAP}_{\text {Scargle }}=1-[1-\exp (-z)]^{N_{i}} \cdot N_{i}$ is the number of independent frequencies. For evenly spaced data, $N_{i}$ can be estimated as $N_{i}=-6.362+1.193 N+0.00098 N^{2}$, where $N$ is the number of datapoints (Horne \& Baliunas 1986). For "clumped" data, however, $N_{i}$ (and with it the FAP Scargle $_{\text {) will }}$ decrease drastically. For a first estimate of the FAP $_{\text {Scargle, we }}$ use $N_{i}=N / 2$. Later, we will determine reliable empirical False Alarm Probabilities for the detected periods.

Periodograms are always contaminated with "false" peaks, namely sidelobes and aliases, caused by the windowing of the data. The recorded time series is the convolution of the signal with this window function. Thus, any period search based on the raw periodogram alone will deliver spurious detections. To circumvent this problem, we used the CLEAN algorithm by Roberts et al. (1987), which deconvolves "dirty" spectrum and window function and thus "cleans" the periodogram. This algorithm is therefore able to distinguish between real peaks and spurious features in the periodogram. Since the CLEAN algorithm is based on the classical periodogram, it does not allow an a priori estimation of the FAP. The combination of Scargle periodogram and CLEAN algorithm, however, delivers reliable period detections and a FAP estimate. Therefore, the period search procedures of numerous recent variability studies rely exclusively on these two techniques (e.g., Bailer-Jones \& Mundt 1999, 2001; Terndrup et al. 1999; O’Dell et al. 1997; Patten \& Simon 1996).

As an additional test to control the significance of periods independently from periodograms, we propose here a method based on the F-test. If a detected period is not significant, then the variance of the time series without the period should be not significantly different from the variance with that period. We fitted each detected period with a sine wave and compared the scattering of the original time series $\sigma$ with that of the residuals of the fit $\sigma_{\text {sub }}$. From $F=\sigma^{2} / \sigma_{\text {sub }}^{2}$ follows the probability that the two variances are equivalent. Thus, this FAP $_{\mathrm{F}-\text { test }}$ indicates, how probable it is that the found period is caused by chance variations in the noise of the photometry (and not in the periodogram). Our results (see Figs. 7 and 8) show that the sine wave approximation is reasonable at least for low signalto-noise, i.e. for objects for which reliable FAP determination is critical. It turns out that the $\mathrm{FAP}_{\mathrm{F}-\text { test }}$ is in all cases larger than the FAP $_{\text {Scargle }}$.

As a first step in our final period search procedure, we filtered the lightcurves to exclude obvious outliers. The following criteria must be fulfilled to accept a period. 

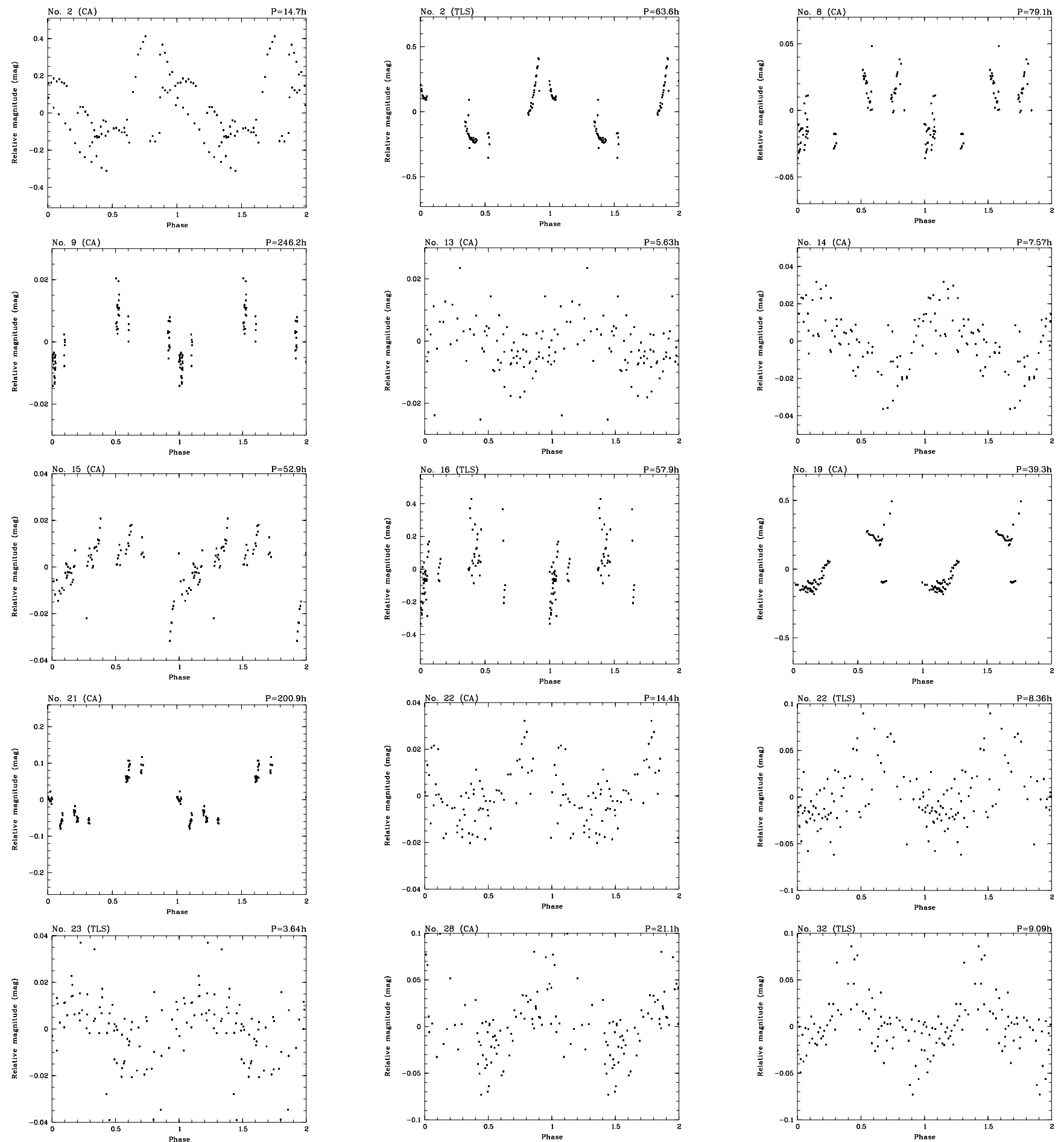

Fig. 7. Phased lightcurves for the detected periodicities. No. and period from Tables 3 and 4 are indicated.

- The Scargle periodogram has a significant peak, i.e. with FAP $_{\text {Scargle }}<1 \%$;

- the Scargle periodograms of at least ten near neighbour stars show no significant peak at a similar frequency, i.e. no corresponding period is within $\pm 10 \%$ of the candidate period;

- phased lightcurve and the original lightcurve itself show the period clearly;
- the variance of the original lightcurve is significantly different from the variance of the lightcurve without the (sine wave approximated) period, i.e. $\mathrm{FAP}_{\mathrm{F}-\text { test }}<10 \%$;

- the lightcurves of nearby stars phased to the candidate period show no significant periodicity;

- the CLEAN algorithm does not reject the period as spurious feature in the periodogram. 

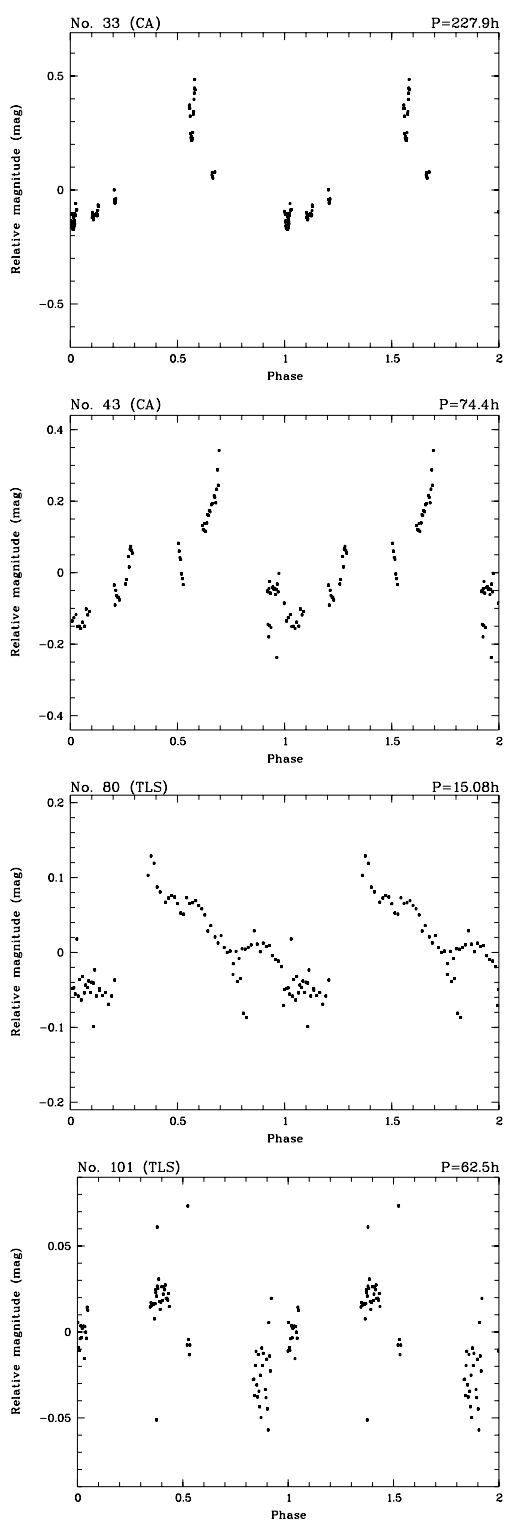
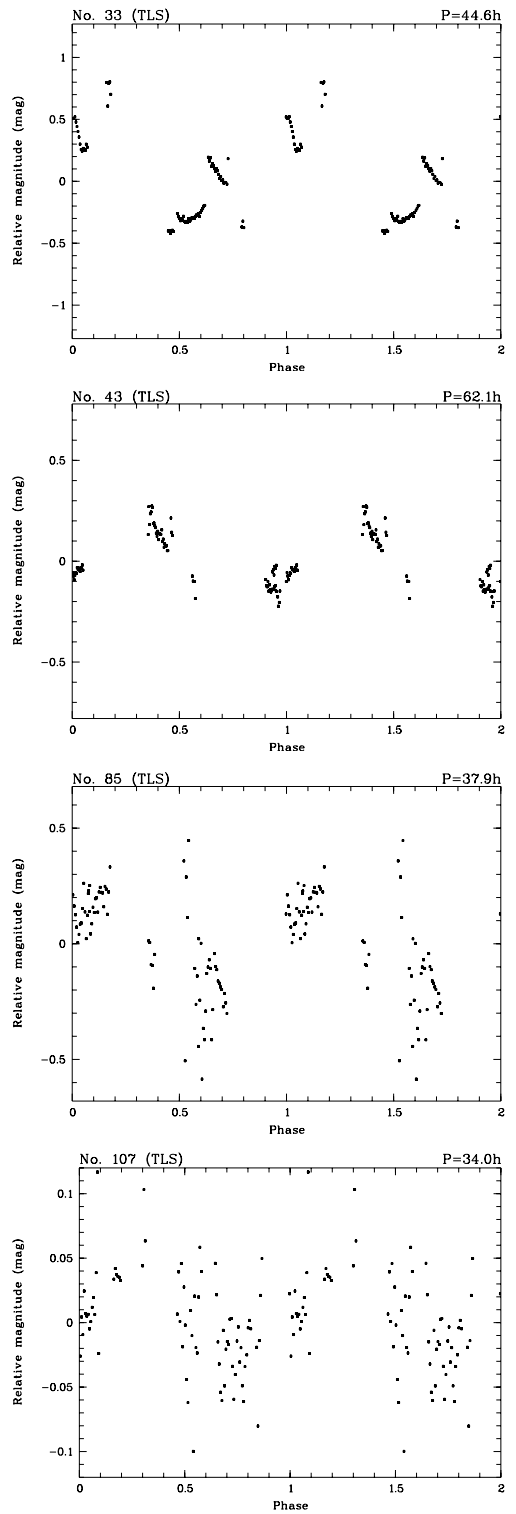
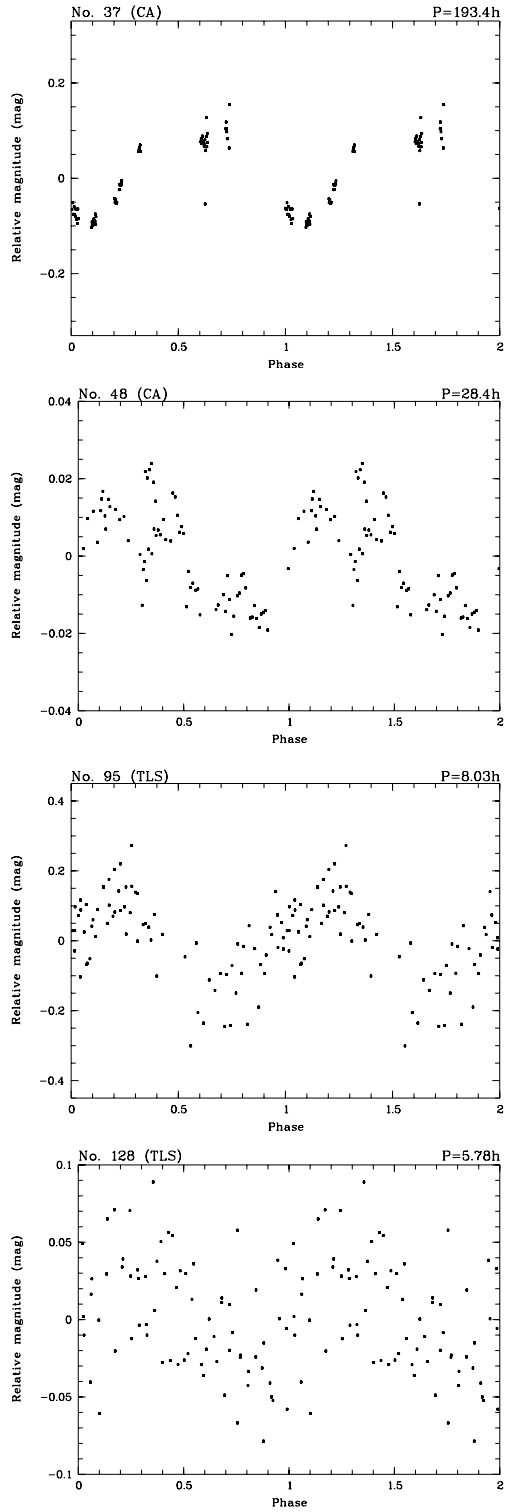

Fig. 8. Phased lightcurves for the detected periodicities (continued).

We established periodic variability for 14 CA and 13 TLS targets. Periods range from 4 to $240 \mathrm{~h}$, amplitudes from 0.02 to 1.1 mag. Tables 3 and 4 list the relevant data for all objects with periodic variability. Figures 7 and 8 show the lightcurves phased to the measured period.

To determine reliable FAP for the periodicities, we used a bootstrap approach as proposed e.g. by Kürster et al. (1997). The resulting empirical FAP will be called $\mathrm{FAP}_{\mathrm{E}}$ in the following. We obtained 10000 randomized data sets with the same sampling as the original lightcurve by retaining the observing times and randomly redistributing the relative magnitudes amongst the observing times. We calculated the Scargle periodogram for each of these data sets and recorded the power of the highest peak. The $\mathrm{FAP}_{\mathrm{E}}$ is the fraction of data sets for which the power of the highest peak exceeds the power of our detected periodicity. This time-expensive simulation must be accomplished for every object on its own to account for the slightly different window function caused by our filtering.
Therefore, we did not include these simulations in the period search criteria outlined above, and used instead the FAP $_{\text {Scargle }}$ and FAP $_{\mathrm{F} \text {-test }}$ as preliminary significance test. It turned out, however, that all detected periods were indeed highly significant with FAP $_{\mathrm{E}}<1 \%$. Although the estimated FAP $\mathrm{P}_{\text {Scargle }}$ tend to be somewhat higher than the $\mathrm{FAP}_{\mathrm{E}}$, the two values are consistent within the statistical uncertainties of the simulation.

It should be noted that the described bootstrap simulation possibly delivers optimistic values for the FAP. Young stars frequently show irregular variability on timescales of hours to days, and it is likely that some of our targets exhibit a similar behaviour. Therefore, Herbst \& Wittenmyer (1996) discussed that datapoints collected within one night may not be independent. On the other hand, our bootstrap algorithm works on uncorrelated data sets and makes no attempt to simulate possible irregular variations. Since the data sets are produced by "shuffling" the original lightcurve, such variations are taken into account as increased noise. 
Table 3. Candidates with significant periodic variability in the CA campaign. Periods $(P)$ are determined by fitting the CLEANed periodogram peak with a Gaussian. Period errors $(\Delta P)$ are based on the half width at half maximum of the periodogram peak, transformed in time space. The amplitudes $(A)$ correspond to the peak-to-peak-range of the binned lightcurve. $N$ is the number of datapoints used for the period search.

\begin{tabular}{rcccccc}
\hline \hline No. & $M\left(M_{\odot}\right)$ & $P(\mathrm{~h})$ & $\Delta P(\mathrm{~h})$ & $A(\mathrm{mag})$ & FAP $_{\mathrm{E}}(\%)$ & $N$ \\
\hline 2 & 0.07 & 14.7 & 0.26 & 0.385 & $<0.01$ & 71 \\
8 & 0.10 & 79.1 & 12.0 & 0.045 & $<0.01$ & 71 \\
9 & 0.17 & 246 & 102 & 0.017 & $<0.01$ & 71 \\
13 & 0.77 & 5.63 & 0.05 & 0.024 & 0.13 & 76 \\
14 & 0.17 & 7.57 & 0.09 & 0.040 & $<0.01$ & 72 \\
15 & 0.20 & 52.9 & 4.24 & 0.034 & $<0.01$ & 71 \\
19 & 0.65 & 39.3 & 2.34 & 0.397 & $<0.01$ & 77 \\
21 & 0.67 & 201 & 74.4 & 0.147 & $<0.01$ & 76 \\
22 & 0.06 & 14.4 & 0.40 & 0.035 & $<0.01$ & 69 \\
28 & 0.03 & 21.1 & 0.65 & 0.057 & $<0.01$ & 71 \\
33 & 0.17 & 228 & 86.4 & 0.454 & $<0.01$ & 72 \\
37 & 0.70 & 193 & 66.3 & 0.192 & $<0.01$ & 78 \\
43 & 0.06 & 74.4 & 8.25 & 0.318 & $<0.01$ & 72 \\
48 & 0.11 & 28.4 & 1.25 & 0.028 & $<0.01$ & 71 \\
\hline
\end{tabular}

Kearns et al. (1997) and Stassun et al. (1999) circumvent the problem by determining the FAP with Monte-Carlo simulations based on synthetic lightcurves of noise with two dispersions, one representing the variations during one night and the other representing the night-to-night variability, calculated as the standard deviation of nightly means. This simulation, however, does not adequatly reproduce intrinsic irregular variability, because even periodic variations will cause an increased night-to-night scatter. Hence, it is not guaranteed that the synthetic lightcurves contain no periodic component. Therefore, the method will overestimate the FAP. Moreover, the method assumes a priori that the datapoints are normally distributed and that the timescale for intrinsic fluctuations is one day. A third drawback of the method emerges when the observing conditions are highly variable and there are nights with very few datapoints, like in our case. Under these circumstances, the night-to-night scatter (as defined above) is determined with high uncertainty. For all these reasons, we consider this approach not applicable to our data.

Another possibility to mimic intrinsic night-to-night variability in simulated lightcurves is to redistribute only the integer part of the observing dates randomly (Herbst et al. 2002). This approach, however, delivers test lightcurves with significantly different sampling than the original time series, a serious drawback, because the FAP depends critically on the window function. Moreover, this procedure only makes sense for time series with datapoints from many observing nights, and is therefore not applicable to our data. Lamm et al. (2004) compare the FAP from bootstrap simulations with and without taking into account the correlations between the datapoints. They found that the Scargle power for a FAP of $1 \%$ decreases only
Table 4. Candidates with significant periodic variability in the TLS campaign. Columns as in Table 3.

\begin{tabular}{rcccccc}
\hline \hline No. & $M\left(M_{\odot}\right)$ & $P(\mathrm{~h})$ & $\Delta P(\mathrm{~h})$ & $A(\mathrm{mag})$ & FAP $_{\mathrm{E}}(\%)$ & $N$ \\
\hline 2 & 0.07 & 63.6 & 8.14 & 0.547 & $<0.01$ & 75 \\
16 & 0.03 & 57.9 & 7.41 & 0.523 & $<0.01$ & 74 \\
22 & 0.06 & 8.36 & 0.13 & 0.068 & $<0.01$ & 76 \\
23 & 0.19 & 3.64 & 0.03 & 0.027 & $<0.01$ & 74 \\
32 & 0.04 & 9.09 & 0.16 & 0.071 & $<0.01$ & 76 \\
33 & 0.17 & 44.6 & 3.79 & 1.114 & $<0.01$ & 77 \\
43 & 0.06 & 62.1 & 7.33 & 0.314 & $<0.01$ & 77 \\
80 & 0.17 & 15.1 & 0.44 & 0.173 & $<0.01$ & 76 \\
85 & 0.02 & 37.9 & 2.66 & 0.468 & $<0.01$ & 74 \\
95 & 0.02 & 8.03 & 0.11 & 0.283 & $<0.01$ & 76 \\
101 & 0.26 & 62.5 & 7.37 & 0.049 & $<0.01$ & 75 \\
107 & 0.27 & 34.0 & 2.20 & 0.097 & 0.07 & 75 \\
128 & 0.02 & 5.78 & 0.07 & 0.066 & 0.39 & 73 \\
\hline
\end{tabular}

marginally when the simulation relies on uncorrelated datapoints. We conclude that our $\mathrm{FAP}_{\mathrm{E}}$ might be optimistic values, but since the $\mathrm{FAP}_{\mathrm{E}}$ for all our periods is below $0.4 \%$ (and for $89 \%$ of our periods even below $0.01 \%$ ), we are nevertheless confident about their significance. Finally, we note that every FAP can only be treated as a relative value, only comparable with values derived with exactly the same procedure.

\subsection{Sensitivity and completeness}

The sensitivity range of the period search is determined by our time sampling. For regularly spaced lightcurves, the upper frequency limit - and thus the lower period limit $P_{\min }$ - is given by the Nyquist frequency $v_{\max }=\frac{1}{2 \Delta}$, where $\Delta$ is the (constant) distance between two datapoints. The upper period limit $P_{\max }$, on the contrary, corresponds to the overall length of the time series. For irregularly spaced data, these relations are only a rough approximation. For a reliable determination of $P_{\min }$ and $P_{\max }$, we carried out a simulation: for both campaigns, we selected a number of field stars which are non-variable according to the generic variability test described in Sect.4.1. Periods from 0.1 to $300 \mathrm{~h}$ were added as pure sinewaves to the lightcurves of these objects. The amplitudes of the test periods were chosen so that the signal-to-noise ratio (SNR, defined as ratio between amplitude of periodicity and scatter in the original lightcurve) is larger than 2 . For each period, the frequency of the highest peak in the Scargle periodogram was recorded. The difference between imposed and detected period delivers an estimate for the reliability of the period search for this period.

Figure 9 shows the absolute difference between true and detected period for both campaigns. The dotted line corresponds to a period error of $10 \%$. The simulation shows that the sensitivity varies with the period. We define $P_{\max }$ as the longest period for which a detection with error below $10 \%$ is possible and obtain $90 \mathrm{~h}$ for the TLS and $270 \mathrm{~h}$ for the CA dataset. Below this limit, there exist several, usually narrow windows 

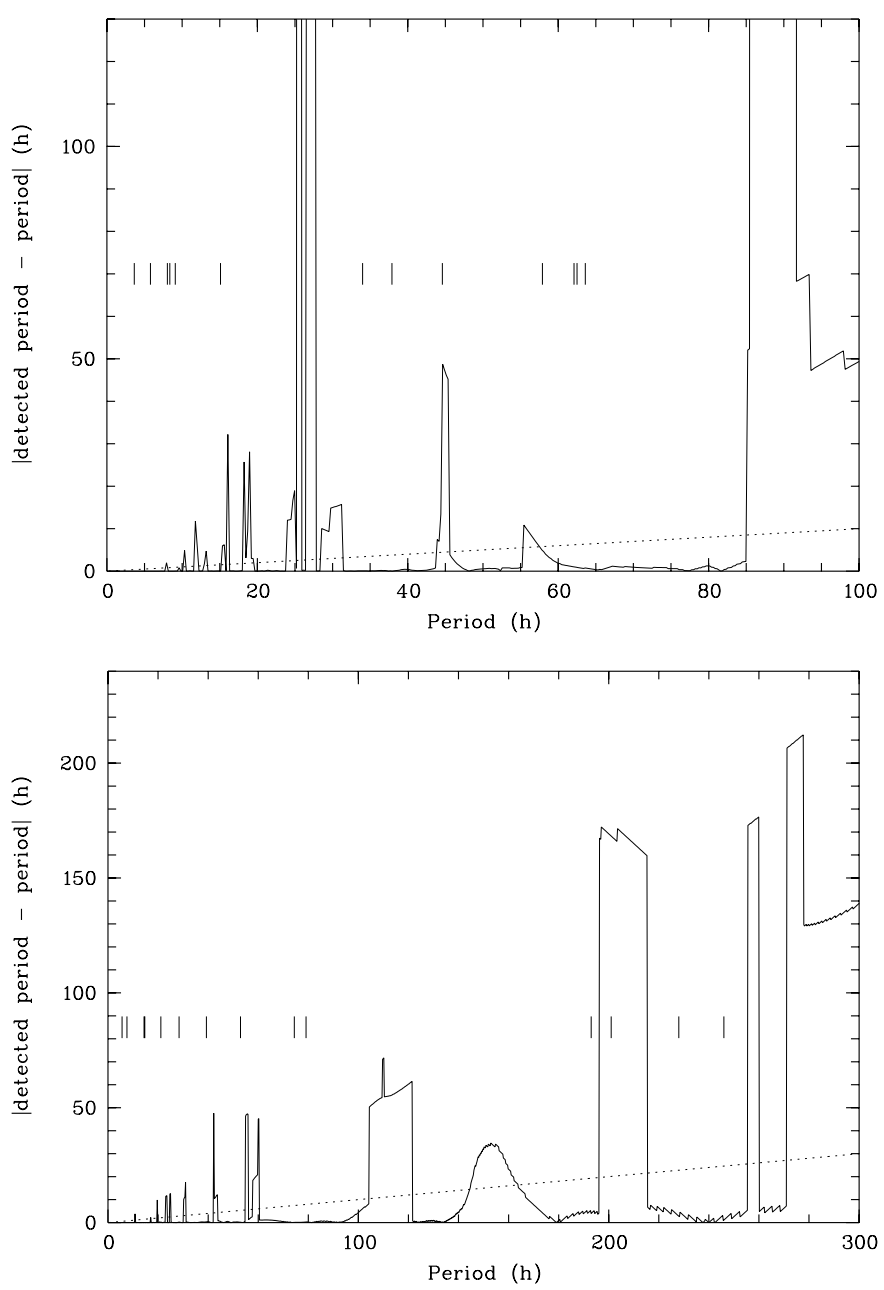

Fig. 9. Sensitivity of the period search: the absolute difference between detected period and true period vs. true period for the TLS (upper panel) and the CA run (lower panel). The signal-to-noise ratio of the shown periodicity is 5 ; the dotted line corresponds to a period error of $10 \%$. The vertical bars indicate the periods found with our time series analysis.

where the period search with the Scargle periodogram is not reliable. These windows are caused by gaps in the datapoint distribution shown in Fig. 4. Also for short periods the reliability of the period search fluctuates somewhat. This illustrates the commonplace that for non-continuous time series the period search will never be complete. In Fig. 9, we also show the periods found with our time series analysis (see Tables 3 and 4) as vertical bars. With one exception, all periods fall in regions where the period determination is reliable. The only exception, the $200 \mathrm{~h}$ CA period for object No. 21, will be discussed below.

On the other hand, the simulation shows that it is easily possible to detect periods down to the Nyquist limit (which is $\approx 0.5 \mathrm{~h}$ for both runs) and below. This is in agreement with Eyer \& Bartholdi (1999) who show that for time series with irregular spacing the Nyquist limit is only an upper limit for $P_{\min }$. These results are more or less independent of the SNR, at least for values $>2$, in agreement with similar simulations by Wolk (1996). For the simulation shown in Fig. 9, we used a periodicity with SNR of 5, a value typical for our periodicities. For a particular target, this plot can look slightly different. When the SNR of its lightcurve is very high, the width and heigth of the low-sensitivity windows will be slightly decreased. The $200 \mathrm{~h}$ period for CA target No. 21 (see Fig. 7) falls into such a window. Since the lightcurve of this object has a SNR of 10, much higher than the SNR of 5 for which Fig. 9 was computed, we consider this detection to be reliable within the given errors (see Table 3 ).

\subsection{Pooled variance diagrams}

The period analysis described in Sect. 4.2 detected periodic variability in 27 lightcurves. Some of these lightcurves, however, clearly show variability on timescales different from the adopted rotation period. Hence, the corresponding phase diagrams show additional variability superimposed on the adopted period. Moreover, some of the targets also show different periods in both runs, as we will outline in Sect. 4.5. To verify the period search results, in particular for the objects where the phase plots are less convincing, we therefore investigated the pooled-variance diagram (PVD) algorithm, which was introduced by Dobson et al. (1990). The basic approach of this method is to divide the time series into equal bins of specified length and to calculate the variance for each bin. The mean of these values (the pooled variance) measures the variability on the specified timescale. If the time series contains a period, the pooled variance should show a plateau around this period, starting roughly at $P / 2$. Although the PVD is surely not suitable for precise period determination, it allows a robust verification of the found periods. Moreover, it will reveal if there is substantial evidence for variability on timescales different from the period. We used a PVD implementation from Kürster, described in detail in Kürster et al. (2000). The program was applied to all lightcurves with detected periodic variability. Especially interesting examples for the resulting PVD are shown in Fig. 10.

The most important result of our PVD analysis is the confirmation of the periods given in Tables 3 and 4 . There is no case where the PVD shows no plateau around the adopted period. According to the PVD, the objects with detected periodicity clearly fall into two groups. The PVD for targets with lowamplitude variability reveal a broad plateau around the period, as shown in the upper left panel of Fig. 10. Sometimes there is an additional plateau at timescales comparable with the overall length of our lightcurves, which can be attributed to long-term variations. On the other hand, objects with high photometric amplitude in most cases show several plateaus (see the upper right panel of Fig. 10 for an example). Apart from a clear flat area around the adopted period, there are often plateaus between 0.2 and 1.0 days, which cannot be attributed to periodicities. Thus, for these objects the PVD indicate the existence of short-term variability superimposed to the period.

The objects Nos. 2, 33, and 43 show high amplitude variations in both campaigns. In the following, we discuss exemplarily the PVD for object No. 2. In the lower part of Fig. 10, we show the PVD for the CA (left panel) and the TLS (right panel) lightcurve. The CA lightcurve clearly shows a plateau around the adopted period of $14.7 \mathrm{~h}$. There is also evidence for variability on even shorter timescales, but no signs for 

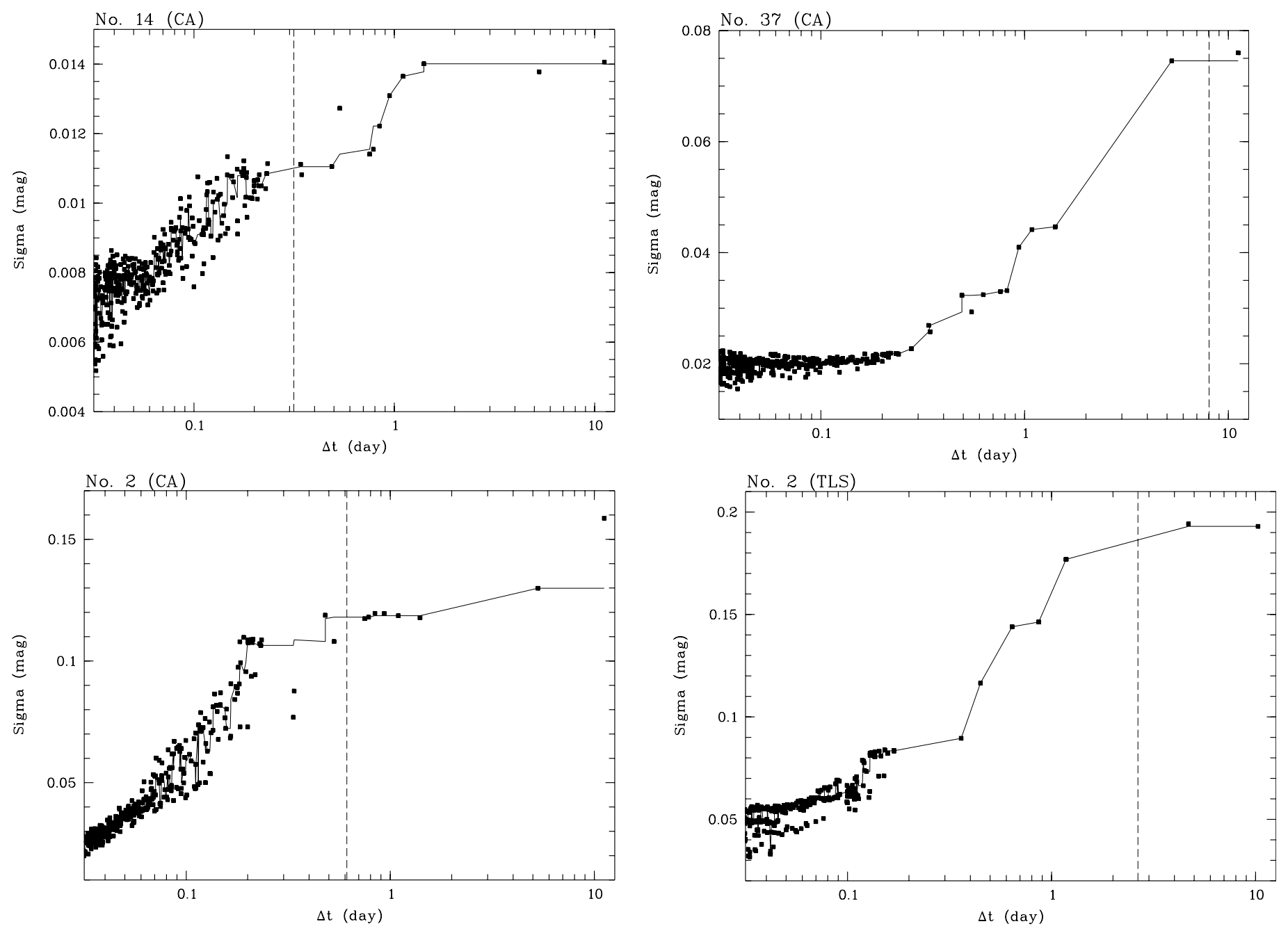

Fig. 10. Pooled variance diagrams for selected lightcurves. The solid lines show a running median over five datapoints. The periods adopted in Tables 3 and 4 are indicated as dashed line.

a period of $63.6 \mathrm{~h}$, as detected in the TLS lightcurve. The PVD for the TLS data, however, shows several plateaus, including one around the $63.6 \mathrm{~h}$ period and a second around $16 \mathrm{~h}$, in agreement with the period from the CA lightcurve. Hence, only the CA period is confirmed by both diagrams. The same result was obtained for objects Nos. 33 and 43. Since the TLS periodograms deliver ambiguous results, offering two possible periods, of which the CA periodograms confirm only one, which is also in agreement with the PVD analysis, we therefore consider the CA periods of these three targets more likely to be the rotation period.

\subsection{Long-term variability}

The variability analysis was accomplished separately for both campaigns. As mentioned above, 27 objects in the CA field were also contained in the TLS catalogue. Since both time series campaigns were separated by 10 months, it is possible to investigate the long-term variability for those targets that were observed twice. The strongly different noise characteristics of both runs, however, complicates the comparison of the analysis results.
First, we compare the generic variability test results from Sect.4.1. Five objects (the high-amplitude objects Nos. 2, 33, 43 and the low-level variables Nos. 14, 51) are variable in both runs. The remaining targets with variability detection in the CA lightcurve are either too bright or have too low amplitudes to be detected also in the TLS data. On the other hand, there are no objects which are only variable in the noisier TLS time series. Thus, we conclude that variability on young VLM objects can persist over one year.

We examined whether a detected period is present in the alternative campaign as well. For nine of the doubly-detected targets, we find periodic variability in the CA time series.

- The objects Nos. 2, 33 and 43 show high amplitude variations in both campaigns. As discussed in Sect.4.4, the CA periods can be recovered also from the TLS lightcurves, but not vice versa. Therefore, the CA periods are more likely to be the true rotation periods.

- Targets Nos. 8, 9, 15, 28 show low amplitude periods in the CA lightcurves. None of the four objects exhibits significant variability in the TLS data, neither periodic nor non-periodic. For two of these targets, the CA amplitudes are too low to be detected in the more scattered TLS lightcurves, i.e. the average rms of the TLS lightcurves 


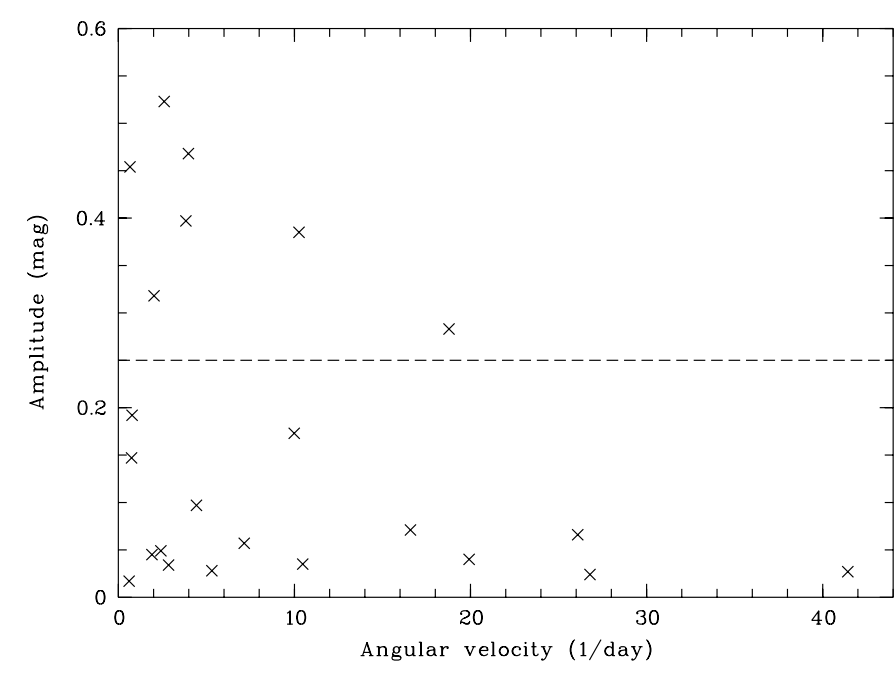

Fig. 11. Angular velocity versus amplitude. The dashed line delineates the separation between low-amplitude and high-amplitude objects.

for the respective I-magnitude is higher than the CA amplitude. However, for targets 8 and 15 the CA amplitude would be detectable even in the TLS lightcurves. Thus, the flux modulation must be significantly reduced compared to the CA data.

- Target No. 14 has an $8 \mathrm{~h}$ period in the CA lightcurve and was significantly variable during the TLS run. However, the lightcurve is slightly contaminated by extinction residuals, possibly preventing the detection of a period.

- Target No. 22 exhibits a significant $8.4 \mathrm{~h}$ period in the TLS lightcurve and a $14.4 \mathrm{~h}$ period in the CA data, i.e. the CA period is roughly twice the TLS period. In the next section, we will attribute the periodic modulations to corotating surface features. It is easily possible that the distribution of surface features produces a flux modulation at half of the rotation period, e.g. through two opposite spots. Therefore, the $14.4 \mathrm{~h}$ period is more probably the true rotation period.

Additionally, three targets with period detection in the TLS run (Nos. 16, 23, 32) are not variable during the CA campaign. This is particularly surprising in the case of No. 16, since it shows high-amplitude variations during the TLS run. The remaining two cases, Nos. 23 and 32, show the inverse behaviour of Nos. 8 and 15 , i.e. their low-amplitude variability is only present in one run and at least diminished in the other.

\section{Interpretation of the variability}

In this section, we argue that the observed variability has its origin in two fundamentally different kinds of surface activity. We discriminate the two types of variability by means of the photometric amplitude, as given in Tables 3 and 4. For periodicities detected in the TLS and the CA lightcurve, we use the CA amplitude in the following. In Fig. 11 we plot amplitude vs. angular velocity $(\Omega=2 \pi / P)$ for all variable objects. Clearly, there is a strong cumulation of targets with low-level variability. The remaining objects show variability with amplitudes ranging from 0.28 to $0.55 \mathrm{mag}$. There is a clear gap of around $0.1 \mathrm{mag}$ width around amplitudes of $0.25 \mathrm{mag}$, indicated by a dashed line in Fig. 11. Furthermore, the high-amplitude lightcurves deviate visibly from a strict sine shape. To quantify these deviations, we fitted all lightcurves with a sine function and measured the rms of the residuals. If the variability is wellapproximated with a sine fit, the rms should not significantly exceed the mean rms of field stars, as given in Figs. 5 and 6 (solid lines). It turns out that most of the highly variable objects with amplitudes $>0.25$ mag still show considerable variability after the sine wave has been subtracted, discriminating them clearly from the low-amplitude objects. The classification in "low-amplitude" and "high-amplitude" variables is also confirmed by the pooled variance diagrams (Sect.4.4), since only the highly variable objects show clear signs of variability on timescales different from the rotation period. In the following, we discuss possible origins for the low-level and the high-level variability.

\subsection{Low-level variability}

As shown above, we established periodic variability with amplitudes $<0.2$ mag for 16 objects. Similar periodicities are wellknown for stars in young open clusters (e.g., Krishnamurthi et al. 1998; Patten \& Simon 1996). Such variability can be explained by the existence of asymmetrically distributed surface features co-rotating with the objects.

Our variable targets have effective temperatures of $>2700 \mathrm{~K}$, estimated from the 3-Myr-isochrone of Baraffe et al. (1998). Hence, they are probably too hot to harbour condensated dust clouds in their atmospheres (see Allard et al. 2001). The origin of the low-level variability are therefore most probably magnetically induced spots, slightly cooler or hotter than their photospheric environment. The properties of these spots, i.e. temperature and filling factor, can only be determined from multi-filter/spectroscopic monitoring or future Doppler imaging campaigns. Nonetheless, our results give first clues about photospheric surface activity on very young VLM objects. Only $17 \%$ of the targets show periodic variability - a significantly lower rate than in similar studies for solar mass stars, indicating low activity levels. This is contrary to the results of the X-ray study by Mokler \& Stelzer (2002), who find no decrease of X-ray activity with mass. Their sample of X-ray detections could, however, be biased against objects with ongoing accretion. Thus, at least part of the X-ray flux is possibly not caused by photospheric surface activity.

There are only two objects which show low-level variability in both campaigns (Nos. 14 and 22). For these two objects no unambiguous period could be found. For object No. 22, this can be explained by evolving spot distributions (see Sect. 4.5). On the other hand, there are four variable objects whose flux modulation must be significantly reduced in one of the campaigns compared to the other, i.e. we find that the activity level of $50 \%$ of our objects has significantly changed in the course of 10 months. Thus, these results give first evidence for the evolution of spot activity on young VLM objects.

\subsection{High-level variability}

Apart from the low amplitude periodic objects, there are 7 additional objects whose lightcurve amplitudes are $>0.25 \mathrm{mag}$ 


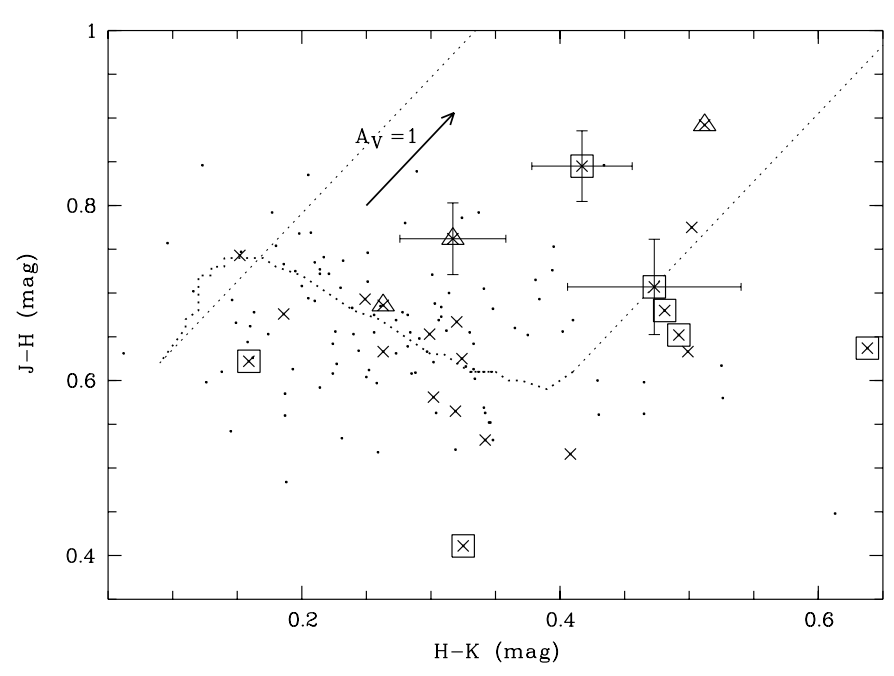

Fig. 12. $(H-K, J-K)$ colour-colour diagram for the cluster member candidates in our field. Crosses are periodically variable objects. Overplotted symbols flag objects with high photometric amplitude: squares mean that the lightcurve amplitude exceeds $0.25 \mathrm{mag}$, and thus mark the objects defined as high-amplitude targets in Sect. 5. Triangles are assigned to transition objects with lightcurve amplitudes between 0.1 and $0.25 \mathrm{mag}$. The solid line indicates the $3 \mathrm{Myr}$ isochrone from the Baraffe et al. evolutionary tracks. Dotted lines show the interstellar extinction vector calculated from Mathis (1990). A reddening vector for $A_{\mathrm{V}}=1 \mathrm{mag}$ is given. For some reddened objects, we overplot the errorbars for the photometry.

and reach up to $1.1 \mathrm{mag}$. The periodograms of their lightcurves show several highly significant peaks. After fitting these periods with a sine wave and subtracting the fit, significant residuals with timescales of hours remain in most cases. The amplitude of these irregular residuals is strongest for targets Nos. 2, 33 and 43 . We conclude that the signal of these targets consists of high-amplitude periodic variations, probably corresponding to the rotation period, and superimposed, sporadic, short-term features. The pooled variance diagrams for highly variable objects imply that the timescale for these irregular variations is $0.2 \ldots 1.0$ days (Sect. 4.4).

It is not possible to explain this high-level variability by the usual scenario of photospheric spots alone: to cause a flux variation of $0.75 \mathrm{mag}$, we would need a zero-emission spot with half the size of the visible hemisphere - this is physically impossible. Eclipse events deliver no plausible interpretation as well, since they should cause strictly periodic light changes. Thus, we are confronted with a variability characteristic not known before for VLM objects.

As outlined in Sect. 1, however, the observed variability characteristic is very similar to the high-level photometric variations of classical T Tauri stars (CTTS). Several groups obtained lightcurves of young solar-mass stars which show a) periodic variability on timescales of days and b) irregular variations on timescales of hours (e.g., Herbst et al. 2000; Fernández \& Eiroa 1996; Bouvier et al. 1995). This behaviour is usually explained by the existence of hot spots formed by matter flowing from an accretion disk onto the stellar surface. Short-term variations can then be understood as a consequence of accretion rate variations and disk instabilities. Our photometry shows that the photometric behaviour of CTTS extends far down into the substellar regime, without obvious mass dependence. This implies the existence of $\mathrm{T}$ Tauri-like accretion disks around VLM objects.

A widely used method to check for the existence of circumstellar disks is the comparison of the near-infrared colours with the intrinsic colours of the targets (e.g., Edwards et al. 1993). A disk would manifest itself by a significant near-infrared excess. Figure 12 shows the $(H-K, J-K)$ colour-colour diagram for the cluster member candidates, constructed from 2MASS data (see Table 7). The solid line indicates the $3 \mathrm{Myr}$ isochrone from the Baraffe et al. (1998) evolutionary tracks. Periodically variable objects are marked with a cross. Overplotted squares are assigned to highly variable objects with amplitudes $>0.25 \mathrm{mag}$, triangles to transition objects with amplitudes between 0.1 and $0.2 \mathrm{mag}$. Most of the non-variable cluster members are distributed symmetrically around the isochrone, where the scatter is determined by the photometric errors, reconfirming that the extinction towards $\sigma$ Ori is negligible and uniform over the cluster area (see also Béjar et al. 1999, 2001). The periodic variable objects with low amplitude populate (with two exceptions) the same region in the diagram.

The highly variable objects, in contrast, clearly tend to lie on the red side of the isochrone, down or even outside the reddening band (dotted lines). Spectroscopy for three of these objects assures their membership of the VLM population around $\sigma$ Ori (see Sect. 7.1). The fact, however, that the vast majority of the $\sigma$ Ori members only scatter around the isochrone shows that the reddened objects must suffer from intrinsic reddening. The fraction of objects with near-infrared excess (i.e. more than $1 \sigma$ reddening) is $71 \%$ for high amplitude and $66 \%$ for transition objects, but $15 \%$ for low amplitude targets. Thus, we conclude that most of the highly variable objects exhibit a near-infrared excess, indicating that they are surrounded by an accretion disk. This may also apply to the transition objects. Figure 12 shows that some accreting objects might exist among low amplitude and non-variable targets as well.

The results from Sect. 4.5 show that the high-level variations are persistent over nearly one year, although with significantly changed amplitude. The only exception, object No. 16, with high-level variability in the TLS and not in the CA lightcurve, can be understood if it had a reduced amplitude, since this object has $I=18.8 \mathrm{mag}$ and the mean rms at this brightness is $0.15 \mathrm{mag}$ in the CA time series. Thus having shown the permanence of high-amplitude variability, these targets are the first photometrically selected sample of strongly accreting VLM objects.

Based on this conclusion, we can estimate disk frequencies and lifetimes. Out of 135 cluster member candidates, 7 show high-amplitude variations and thus probably possess an accretion disk. This corresponds to a fraction of 5 to $7 \%$ (taking into account a field star contamination of up to $30 \%$ of our VLM sample). Since we might have missed accreting objects without strong photometric variability, this should be considered as a lower limit. For comparison, Barrado y Navascués et al. (2003), Oliveira et al. (2002), and Barrado y Navascués \& Martín (2003) estimate disk frequencies of $5-14 \%$ for VLM objects in the $\sigma$ Ori cluster. Based on a very small sample of six Brown Dwarfs, Jayawardhana et al. (2003) derived a disk 
frequency of $33 \pm 24 \%$. Given the statistical uncertainties, all these results are in good agreement with our estimate. Thus, it seems that disks are rare in the $\sigma$ Ori cluster, but they exist for VLM stars as well as for Brown Dwarfs. The fact that we do not observe totally irregular variability with high amplitudes is a further indication for the scarcity of strong accretors among VLM objects in the $\sigma$ Ori cluster, since such variations are believed to be typical for the most actively accreting $\mathrm{T}$ Tauri stars in younger clusters (e.g., Herbst et al. 2000).

The low disk frequency for VLM objects in the $\sigma$ Ori cluster implies that most of these objects dissipate their disks before they reach the age of this cluster. Since the most probable age for the cluster is $3 \mathrm{Myr}$ (Zapatero Osorio et al. 2002), the disk lifetime for VLM objects, defined as the timescale for essentially all the stars to lose their disk (Haisch et al. 2001), is roughly 3-4 Myr. In comparison, solar-mass stars retain their disks significantly longer (Haisch et al. 2001), their disk lifetime is $6 \mathrm{Myr}$. Thus, if the age estimate for the $\sigma$ Ori cluster is valid, disk dissipation timescales are shorter in the VLM regime. This is confirmed by a recent study of Oliveira et al. (2003), who investigated near-infrared colours of a sample of $\sigma$ Ori cluster members with $J<13.3$ mag (corresponding to $M>0.2 M_{\odot}$ ). They derived an overall disk frequency of $46-54 \%$, but the near-infrared excess shows a strong decline for $K>11 \mathrm{mag}$, corresponding to $M<0.5 M_{\odot}$ (see their Fig. 5). Thus, most of the stars with disks have solarlike masses, and the disk frequency is decreasing with mass.

\section{Rotation periods}

As discussed above, we attribute the detected periodicities to a rotational modulation of the emitted flux. In this section, we will analyse the implied rotation periods. We will compare the periods from high-level variable with those for low-level variable objects. We will then discuss the mass dependence of rotation. The period sample for all analyses comprises $14 \mathrm{CA}$ and 9 TLS periods, excluding those TLS periodicities also detected in the CA lightcurves (see also the discussion of these objects in Sect. 4.5). The two rotation periods for $\sigma$ Ori objects (SOri 31 and SOri 33) measured by Bailer-Jones \& Mundt (2001) will be used to underline the results.

\subsection{High amplitude vs. low amplitude}

In Sect. 5 we argue that we observe two kinds of variability, high-level and low-level variations, where the high-level variability is interpreted as a consequence of ongoing accretion processes, as confirmed by near-infrared photometry (Sect. 5.2) and spectroscopy (see Sect. 7). Thus, highly variable objects are very likely surrounded by circumstellar disks. This could have consequences for their rotational behaviour, since magnetic coupling between star and circumstellar disk is believed to be the mechanism responsible to brake the rotation of T Tauri stars. For solar mass stars, there is indeed observational evidence for a correlation between rotation and disk indicators, in the sense that stars with disks tend to be slow rotators (e.g. Edwards et al. 1993; Herbst et al. 2002). However, there exist controversial investigations which find no correlation

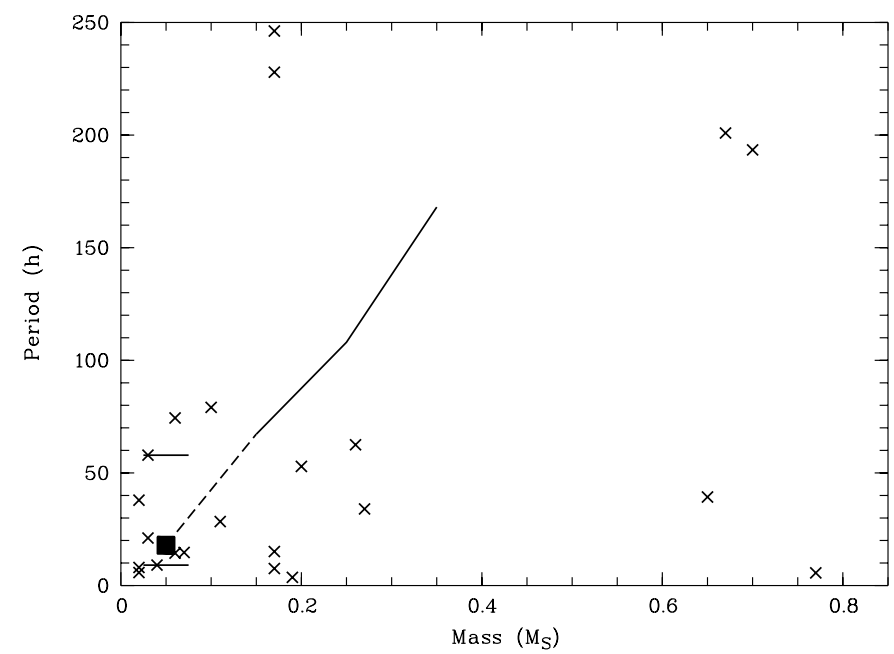

Fig. 13. Mass dependence of the rotation period: plotted are all measured periods from TLS and CA vs. the object mass. The solid line shows the median period published by Herbst et al. (2001). The median for our objects with $M<0.1 M_{\odot}$ is shown as filled square, vertical lines indicate the quartiles (giving an estimate of the statistical uncertainties). This median found for our objects extends the line of Herbst et al. (2001) into the Brown Dwarf regime (dashed line).

between rotation and disk presence (e.g., Stassun et al. 2001), indicating that disk-locking may not be the full solution to angular momentum distribution in young stars (Rebull et al. 2002). Nevertheless, assuming that disk-locking plays a major role as an angular momentum regulation mechanism, we should expect that accreting VLM objects show a rotation period distribution different from the non-accreting ones. In particular, objects with disks should on average rotate more slowly.

In Fig. 11, we plot photometric amplitude vs. angular velocity for all our targets. As argued in Sect. 5.2, the plot shows two populations of datapoints, separated by a gap around amplitudes of $0.2 \ldots 0.25 \mathrm{mag}$, delineated by a dashed line. The objects above this line are probably active accretors. For these objects, the average angular velocity is $\Omega=6.0 d^{-1}$. For amplitudes $<0.25 \mathrm{mag}$, however, $\Omega$ is on average $11.1 d^{-1}$. Thus, low- amplitude objects tend to rotate faster on average, as we would expect for a disk-locking scenario. The periods from Bailer-Jones \& Mundt (2001) are also consistent with this picture, since they are shorter than $10 \mathrm{~h}$ (corresponding to $\Omega>15$ ) and have amplitudes $<0.05$ mag. Hence, the available data may indicate disk-locking processes on VLM objects. Since this result could be affected by low number statistics, we postpone its further discussion until more rotational data are on hand.

It is instructive to compare our rotation periods with those recently published by Joergens et al. (2003). They measured 4 periods for Brown Dwarfs and VLM stars in the ChaI star forming region, which is $1 \mathrm{Myr}$ old and thus considerable younger than the $\sigma$ Ori cluster. All their periods are longer than 2 days. In the context of the disk-locking scenario, these objects could be still disk-locked. This would be in agreement with the large disk frequencies (65\%) among Brown Dwarfs in the similarly-aged Trapezium cluster (Muench et al. 2001). 


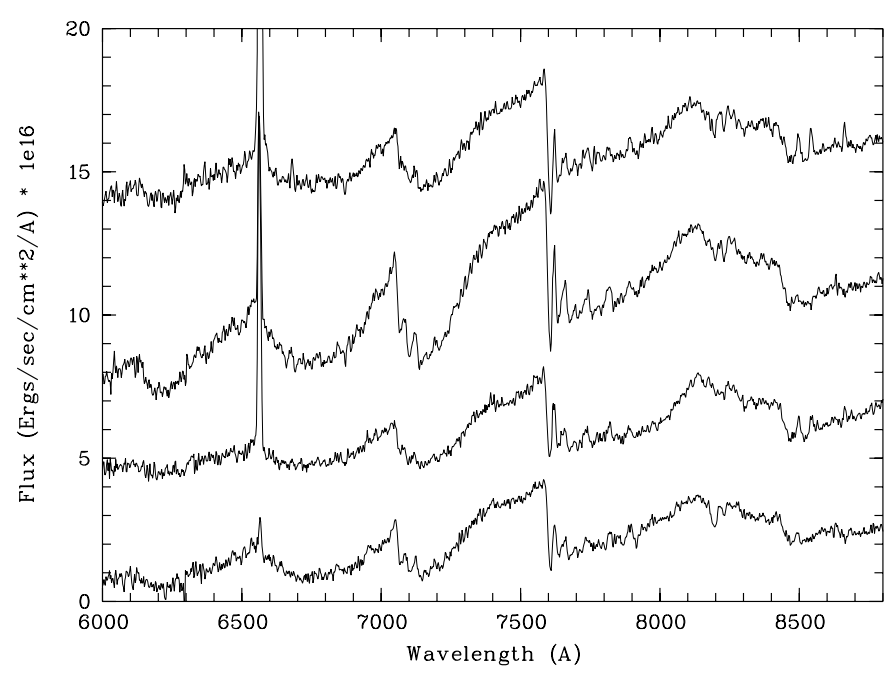

Fig. 14. Optical spectra for three highly variable targets (from top to bottom: Nos. 2, 33, 43) and one non-variable target (No. 90, beneath). The spectra are shifted by 12, 6, 4, 0 (from top to bottom) units for clarity.

\subsection{Mass dependence}

Several groups have reported a bimodal distribution of the rotation periods for very young solar mass stars (e.g. Herbst et al. 2002; Edwards et al. 1993). Since longer periods are significantly correlated with infrared excess attributed to the presence of a circumstellar disk, this is a direct sign for a disk-locking mechanism braking the rotation in the $\mathrm{T}$ Tauri phase. There is observational evidence, however, that the bimodality becomes less obvious for low-mass objects and finally vanishes for objects with masses below $0.2 M_{\odot}$ (Herbst et al. 2001). Our periods show no bimodal distribution throughout the whole mass regime. Since we observed only few targets with masses comparable to the solar mass, the detection of the slow rotator peak in the histogram could be prevented by our low-number statistics. Hence, our data allow no verification of the evolution of bimodality with decreasing mass. Nevertheless, we note that there is definitely no bimodality within our statistical uncertainties for objects with masses in the range from 0.03 to $0.4 M_{\odot}$.

To quantify the mass dependence of the periods, Herbst et al. (2001) calculate a moving median for their periods in the mass regime $0.1 \ldots 0.4 M_{\odot}$, measured for objects in the ONC. They find that lower-mass objects tend to rotate gradually faster. Our results confirm this result. Figure 13 shows masses vs. rotation periods for our $\sigma$ Ori targets. The median period is $46.1 \mathrm{~h}$ for VLM stars, but only $14.7 \mathrm{~h}$ for Brown Dwarfs. Our periods make it possible for the first time to extend the analysis of Herbst et al. (2001) to masses below their detection limit. Figure 13 shows the mass-period relation of Herbst et al. combined with our datapoint for masses $<0.1 M_{\odot}$. Obviously, our periods nicely extend the mass-rotation relation down into the substellar regime. Because of the statistical uncertainties, however, this needs further confirmation.

Recapitulating, we observe two effects: no bimodality and the tendency of faster rotation towards lower masses. Both could be explained by a decrease of the effectiveness of
Table 5. Comparison of the spectra with models of Allard et al. (2000). The first two columns are the parameters of the best fitting model spectrum. The spectral types follow with the effective temperature scale of Leggett et al. (2000). For comparison, we additionally give the $T_{\mathrm{eff}}$ estimated from photometry alone, presuming cluster membership.

\begin{tabular}{l|rrrr}
\hline \hline No. & 2 & 33 & 43 & $90^{*}$ \\
\hline$T_{\text {eff }}(\mathrm{K})$ & 2800 & 3100 & 2900 & 3000 \\
$\log g$ & 4.0 & 3.5 & 4.0 & 3.5 \\
Spectral type & $\mathrm{M} 5.5$ & $\mathrm{M} 4.0$ & $\mathrm{M} 5.0$ & $\mathrm{M} 4.5$ \\
$T_{\text {eff }}(\mathrm{K})-$ phot & 2930 & 3030 & 2890 & 2850 \\
\hline
\end{tabular}

* Non-variable target observed for comparison.

rotational braking through magnetic coupling to a circumstellar disk. Indeed, there is evidence for decreasing disk lifetimes with decreasing mass, as pointed out in Sect.6.1. Moreover, efficient angular momentum removal, either through magnetic coupling or stellar winds, could also be prevented by low magnetic field strengths, as implied by the low activity level inferred from the small rate of variable objects (see Sect. 5.1).

\section{Accreting VLM objects}

In this section, we concentrate again on the discussion of the highly variable targets, which were identified as actively accreting VLM objects in Sect. 5. To substantiate this interpretation, we obtained low-resolution spectroscopy with MOSCA at the 3.5-m telescope on Calar Alto and grism red500, covering a wavelength range of $5400 \ldots 10000 \AA$ with a dispersion of $2.9 \AA /$ pixel. We observed two Brown Dwarfs and one VLM star with high-level variability in both time series (Nos. 2, 33, 43) and, for comparison, one non-variable Brown Dwarf (No. 90). For relative flux calibration, we additionally obtained a spectrum of the spectrophotometric standard star GD71 (Bohlin et al. 1995). Spectra of $\mathrm{Ar} / \mathrm{ArHg} / \mathrm{Ne}$ arc lamps were used for wavelength calibration. Reduction and calibration was done with the IRAF standard routines within the package onedspec. We note that the flux is considerably damped for $\lambda>8000 \AA$, because of second order overlap in the spectra. All four spectra are shown in Fig. 14.

\subsection{Spectral classification}

The spectra show all the characteristics expected from latetype objects, in particular deep $\mathrm{TiO}$ and $\mathrm{VO}$ bands (Kirkpatrick et al. 1991). We determined $T_{\text {eff }}$ and $\log g$ from the spectra by comparing them with the STARdusty 2000 models of Allard et al. (2000). For this comparison, we used only the spectral range from 6700 to $7700 \AA$, to exclude regions with strong emission lines and avoid the long wavelength end, where our spectra are not properly flux calibrated. The model grid spans $T_{\text {eff }}=2000 \ldots 4000 \mathrm{~K}$ in $100 \mathrm{~K}$ steps and $\log g=3.5 \ldots 5.5$ in steps of 0.5 . To account for varying atmospheric conditions during the observations and for possible continuum excesses as expected for accreting objects, we allowed for a wavelengthindependent scaling and shifting of the models. 
Table 6. Equivalent widths for prominent emission lines. The first column contains name and laboratory wavelength (in $\AA$ ) of the emission line, then we list the equivalent widths for the four targets. Nondetection of the feature is indicated with a dash, "b" means that the line is present, but strongly blended by nearby features.

\begin{tabular}{l|rrrr}
\hline \hline No. & 2 & 33 & 43 & $90^{*}$ \\
\hline [OI] 6300 & $\mathrm{b}$ & 1.1 & $\mathrm{~b}$ & - \\
[OI] 6364 & 0.9 & - & - & - \\
$\mathrm{H} \alpha 6563$ & 104.5 & 21.3 & 103.7 & 10.7 \\
HeI 6678 & 1.8 & 2.4 & - & - \\
[SII] 6716 & 0.23 & 0.39 & - & - \\
[SII] 6731 & 0.38 & 0.32 & - & - \\
OI 8446 & $\mathrm{b}$ & - & - & - \\
CaII 8498 & 4.8 & 0.6 & 6.9 & 3.8 \\
CaII 8542 & 3.8 & - & 5.1 & - \\
CaII 8662 & 2.5 & - & 3.1 & - \\
HI (P9) 9229 & 0.8 & 0.4 & 0.2 & 0.4 \\
\hline
\end{tabular}

${ }^{*}$ Non-variable target observed for comparison.

We calculated the root mean square between each model and the data. It turns out that the rms values strongly depend on $T_{\text {eff }}$, with a clear minimum. On the other hand, the dependency on $\log g$ is much weaker. Therefore, we first determined the effective temperature at which the rms is smallest. From the set of models with this $T_{\text {eff }}$, we then selected the model with the best fitting $\log g$. Visual comparison of the selected model with the data shows good agreement. The results, i.e. $T_{\text {eff }}$ and $\log g$, are listed in Table 5. Spectral types are estimated with the effective temperature scale of Leggett et al. (2000), which is based on essentially the same models as our analysis.

Assuming that the object is indeed a cluster member, a second estimate for $T_{\text {eff }}$ can be obtained by comparing the photometry with the cluster isochrone of Baraffe et al. (1998). This value of $T_{\text {eff }}$ will be either too high or too low, if the target resides in the fore- or background of the cluster. An estimate in agreement with the true $T_{\text {eff }}$, as measured from the spectra, confirms the cluster membership. We cannot expect perfect agreement, however, since this comparison is affected by photometry errors and the grid size of the models. A further complication is of course the large variability of targets Nos. 2, 33 and 43 . It might be that photometry and spectroscopy caught the object in a totally different accretion state. With these considerations in mind, all objects show good agreement between spectroscopic and photometric effective temperatures, confirming them als VLM cluster members of $\sigma$ Ori. The youth of all four targets its also confirmed by the low surface gravity values. Evolved objects with comparable masses exhibit $\log g>5.3$ (Baraffe et al. 1998), whereas we derived $\log g=3.5 \ldots 4.0$, in agreement with the values expected for a $3 \mathrm{Myr}$ object with $T_{\text {eff }}$ around $3000 \mathrm{~K}$.

\subsection{Emission-line spectrum}

The dominant spectral feature in all three highly variable objects is the large $\mathrm{H} \alpha$ emission line. The non-variable object has
$\mathrm{H} \alpha$ in emission as well, but with considerably lower intensity. A variety of other emission features is present in the spectra, similar to emission spectra of CTTS. We measured equivalent widths for all prominent emission lines by subtracting a linearly fitted continuum from the line flux (Table 6). Obviously, the highly variable targets, in particular targets Nos. 2 and 43, show much more intense emission features than the comparison object. Target No. 33 seems to be more active than No. 90, judged from the $\mathrm{H} \alpha$ equivalent widths. However, compared to Nos. 2 and 43, $\mathrm{H} \alpha$ and $\mathrm{Ca}$ emission lines are relatively faint. It might be that we caught target No. 33 at the minimum of its lightcurve and thus in a relatively quiet state.

Forbidden lines such as [OI] and [SII] can be attributed to low-density regions, e.g. in stellar winds, and are thus tracers of a mass loss process. On the other hand, features like the $\mathrm{H} \alpha$ emission line and the Ca triplet are clear signs of the accretion process. These lines are also common in outflows. For both features, however, we attribute most of the emission to accretion processes: on the one hand, the ratio between $\mathrm{H} \alpha$ and [SII] in our spectra is much larger than 4, which is the upper limit given for (outflow-dominated) Herbig Haro objects (e.g. Böhm $\&$ Goodson 1997). On the other hand, the ratio of the Ca triplet equivalent widths is close to $1: 1: 1$, whereas the predicted ratio for optically thin emission would be 1:9:5. Similar values are common among strongly accreting CTTS (e.g., Reipurth et al. 1986) and clearly differ from those of Herbig Haro flows. The presence of the He $\lambda 6678$ line is a third indicator of ongoing accretion. We conclude that the emission spectrum of those highly variable objects is dominated by the signature of strong accretion processes. Comparing $\mathrm{H} \alpha$ linewidths and spectral types with the classification scheme of White \& Basri (2003), the highly variable objects belong to the VLM analogues of classical T Tauri stars, while the non-variable object is similar to a weak-line $\mathrm{T}$ Tauri star.

Recapitulating, our low-resolution spectroscopy yields a clear result: our highly variable VLM objects are probably accreting material from a disk and are thus the VLM analogues of CTTS. The T Tauri nature of young VLM objects is supported by recent publications, since spectroscopic signatures of typical $\mathrm{T}$ Tauri behaviour have been detected by other groups (e.g., Jayawardhana et al. 2002; White \& Basri 2003; Barrado y Navascués et al. 2003). The existence of disks around Brown Dwarfs is confirmed by independent observations of Natta \& Testi (2001), Natta et al. (2002), Testi et al. (2002), Apai et al. (2002), Liu et al. (2003), Jayawardhana et al. (2003), and López Martí et al. (2004), mostly via midinfrared excesses. All these findings set strong constraints for possible formation scenarios for VLM objects. In competition with star-like formation mechanisms, it was recently proposed that Brown Dwarfs form preferably in orbits around more massive stars, either in protoplanetary disks (Pickett et al. 2000) or as stellar embryos in multiple stellar systems (Reipurth \& Clarke 2001). Both scenarios need an ejection mechanism to explain the large number of isolated Brown Dwarfs found in stellar clusters and star forming regions. It seems unlikely that the objects maintain a significant fraction of their accretion reservoir during this ejection process. Thus, the current 
observational picture favours star-like formation for VLM stars and substellar objects.

\section{Conclusions}

We present the first photometric variability study for VLM members of the young $\sigma$ Ori cluster. With multi-filter photometry, 135 cluster member candidates were identified, including $90 \%$ VLM objects $\left(M<0.4 M_{\odot}\right)$. This preliminary member list still contains about $30 \%$ contaminating field stars and thus needs spectroscopic verification.

We monitored these targets in two I-band time series in January and December 2001. The first campaign, using the 2-m Tautenburg Schmidt telescope, covered 110 candidates, spanning masses from 0.02 to $0.5 M_{\odot}$. The second time series, obtained with the $1.23-\mathrm{m}$ telescope on Calar Alto, registered 52 targets spanning a mass range from 0.02 to $1.4 M_{\odot}$. 27 objects were observed in both runs. After reduction and relative calibration, we obtained differential lightcurves for all targets. We found that the difference image analysis package from Gössl et al. (2002) improves the precision of the Calar Alto lightcurves by several mmag.

Time series analysis was focused on the period search, but we included a test to detect irregular variations as well. We examined the variability of the targets based on the scattering of the lightcurves and found that the very young targets contain significantly more variable objects than field stars. The fraction of variable objects as well as the variability amplitude does not significantly change over the entire mass range.

We detected periodic variability for 14 CA and 13 TLS targets, with periods spanning from 4 to $240 \mathrm{~h}$. Four targets show a periodicity in both campaigns. The periodically variable candidates clearly fall into two groups: 16 of them show low-level variability which is in most cases well-approximated by a sine wave. We argue that this type of variability is most probably caused by magnetically induced photospheric spots co-rotating with the objects. We see evidence for the spot evolution on VLM objects, since most of these targets show the periodicity in only one campaign.

The lightcurves of the remaining objects have amplitudes $>0.25$ mag and show obvious deviations from the sine shape. Their photometric behaviour is very similar to those of classical $\mathrm{T}$ Tauri stars. The quasi-periodic oscillation is therefore most probably caused by hot spots formed by matter flow from an accretion disk. Thus, our results imply the existence of accretion disks around VLM stars and Brown Dwarfs. The fact that most of the highly variable objects show a near-infrared colour excess confirms this finding. Low-resolution spectroscopy of a subsample of these targets assures their cluster membership and their youth. We find strong emission features, in particular a dominating $\mathrm{H} \alpha$ emission line, characteristic for accreting objects. The extension of T Tauri behaviour to VLM stars and Brown Dwarfs strongly supports star-like formation scenarios for these objects.

Highly variable and thus accreting objects rotate on average more slowly than low-level variable targets. This is expected in terms of a disk-locking scenario, where magnetic coupling between star and disk removes angular momentum during the T Tauri phase. In agreement with previous publications, we find that strong accretors are rare in the $\sigma$ Ori cluster, but they certainly exist among VLM stars as well as Brown Dwarfs. Comparison with studies of younger clusters and more massive stars give evidence that disk dissipation timescales are significantly decreased with decreasing object mass.

In agreement with previous publications, we find a tendency of faster rotation towards decreasing object masses, possibly caused by decreasing effectiveness of angular momentum removal. This could either be explained with shorter disk lifetimes or with weaker magnetic fields, as inferred from the low activity level of our targets.

Acknowledgements. It is a pleasure to acknowledge the constructive cooperation with the WeCAPP team. The successful application of OIS would not have been possible without the intensive support in particular from Arno Riffeser. We are very grateful to D. Roberts who provided an implementation of the CLEAN algorithm. Software and support for the pooled variance analysis were kindly provided by Martin Kürster. We thank Arno Riffeser and Jürgen Fliri for their assistance during the observations on Calar Alto. This work was supported by the German Deutsche Forschungsgemeinschaft, DFG project number Ei 409/11-1. The publication makes use of data products from the Two Micron All Sky Survey, which is a joint project of the University of Massachusetts and the Infrared Processing and Analysis Center/California Institute of Technology, funded by the National Aeronautics and Space Administration and the National Science Foundation.

\section{References}

Alard, C., \& Lupton, R. H. 1998, ApJ, 503, 325

Allain, S. 1994, Thèse de DEA, Univ. Joseph Fourier, Grenoble

Allard, F., Hauschildt, P. H., \& Schwenke, D. 2000, ApJ, 540, 1005

Allard, F., Hauschildt, P. H., Alexander, D. R., Tamanai, A., \& Schweitzer, A. 2001, ApJ, 556, 357

Apai, D., Pascucci, I., Henning, Th., et al. 2002, ApJ, 573, 115

Bailer-Jones, C. A. L., \& Mundt, R. 1999, A\&A, 348, 800

Bailer-Jones, C. A. L., \& Mundt, R. 2001, A\&A, 367, 218

Barrado y Navascués, D., Stauffer, J. R., Briceño, C., et al. 2001, ApJS, 134,103

Barrado y Navascués, D., Bouvier, J., Stauffer, J. R., Lodieu, N., \& McCaughrean, M. J. 2002, A\&A, 395, 813

Barrado y Navascués, D., Béjar, V. J. S., Mundt, R., et al. 2003, A\&A, 404, 171

Barrado y Navascués, D., \& Martín, E. L. 2003, AJ, 126, 2997

Baraffe, I., Chabrier, G., Allard, F., \& Hauschildt, P. H. 1998, A\&A, 337,403

Béjar, V. J. S., Zapatero Osorio, M. R., \& Rebolo, R. 1999, ApJ, 521, 671

Béjar, V. J. S., Martín, E. L., Zapatero Osorio, M. R., et al. 2003, ApJ, 556,830

Bertin, E., \& Arnouts, S. 1996, A\&AS, 117, 393, see also http://terapix.iap.fr/soft/sextractor

Böhm, K.-H., \& Goodson, A. P. 1997, Herbig-Haro flows and the birth of stars, ed. B. Reipurth, \& C. Bertout (Kluwer Acad. Publ.)

Bohlin, R. C., Colina, L., \& Finley, D. S. 1995, AJ, 110, 1316

Bouvier, J., Covino, E., Kovo, O., et al. 1995, A\&A, 299, 89

Carpenter, J. M. 2001, AJ, 121, 2851

Chabrier, G., \& Baraffe, I. 2000, ARA\&A, 38, 337

Clarke, F. J., Tinney, C. G., \& Covey, K. R. 2002, MNRAS, 332, 361 
Dobson, A. K., Donahue, R. A., Radick, R. R., \& Kadlec, K. L. 1990, Cool stars, stellar systems, and the sun, Proc. of the 6th Cambridge Workshop, 132

Edwards, S., Strom, S. E., Hartigan, P., et al. 1993, AJ, 106, 372

Eislöffel, J., \& Scholz, A. 2002, The Origins of Stars and Planets: The VLT View, Proc. of the ESO Workshop 2001, ed. McCaughrean, \& Alves, 219

Eyer, L., \& Bartholdi, P. 1999, A\&AS, 135, 1

Fernández, M., \& Eiroa, C., A\&A, 310, 143

Fernández, M., \& Comerón, F. 2001, A\&A, 380, 264

Gelino, Ch. R., Marley, M. S., Holtzman, J. A., Ackerman, A. S., \& Lodders, K. 2002, ApJ, 577, 433

Gössl, C. A., \& Riffeser, A. 2002, A\&A, 381, 1095

Haisch, K. E. Jr., Lada, E. A., \& Lada, Ch. J. 2001, ApJ, 553, 153

Herbst, W., \& Wittenmyer, R. 1996, A\&AS, 189, 4908

Herbst, W., Maley, J. A., \& Williams, E. C. 2000, AJ, 120, 349

Herbst, W., Bailer-Jones, C. A. L., \& Mundt, R., ApJ, 554, 197

Herbst, W., Bailer-Jones, C. A. L., Mundt, R., Meisenheimer, K., \& Wackermann, R. 2002, A\&A, 396, 513

Horne, J. H., \& Baliunas, S. L. 1986, ApJ, 302, 757

Jayawardhana, R., Mohanty, S., \& Basri, G. 2002, ApJ, 578, 141

Jayawardhana, R., Ardila, D. R., Stelzer, B., \& Haisch, K. E., Jr. 2003, AJ, 126, 1515

Joergens, V., Fernández, M., Carpenter, J. M., \& Neuhäuser, R. 2003, ApJ, 594, 971

Kearns, K. E., Eaton, N. L., Herbst, W., \& Mazzurco, Ch. J. 1997, AJ, 114, 1098

Kirkpatrick, J. D., Henry, T. J., \& McCarthy, D. W. Jr. 1991, ApJS, 77, 417

Krishnamurthi, A., Terndrup, D. M., Pinsonneault, M. H., et al. 1998, ApJ, 493, 914

Kürster, M., Schmitt, J. H. M. M., Cutispoto, G., \& Dennerl, K. 1997, A\&A, 320, 831

Kürster, M., Endl, M., Els, S., et al. 2000, IAUS, 202, 5

Lamm, M. H., Bailer-Jones, C. A. L., Mundt, R., Herbst, W., \& Scholz, A. 2004, A\&A, 417, 557

Landolt, A. U. 1992, AJ, 104, 340

Leggett, S. K., Allard, F., Dahn, C., et al. 2000, ApJ, 535, 965

Liu, M. C., Najita, J., \& Tokanuga, A. T. 2003, ApJ, 585, 372

López Martín, B., Eislöffel, J., Scholz, A., \& Mundt, R. 2004, A\&A, 416, 555

Lucas, P. W., \& Roche, P. F. 2000, MNRAS, 314, 858

Martín, E. L., Zapatero Osorio, M. R., \& Lehto, H. J. 2001, ApJ, 557, 822

Martín, E. L., \& Zapatero Osorio, M. R. 1997, MNRAS, 286, 17

Mathis, J. S. 1990, ARA\&A, 28, 37

Mokler, F., \& Stelzer, B. 2002, A\&A, 391, 1025
Moraux, E., Bouvier, J., Stauffer, J. R., \& Cuillandre, J.-C. 2003 , A\&A, 400, 891

Morisson, J. E., Röser, S., McLean, B., Bucciarelli, B., \& Lasker, B. 2001, AJ, 121, 1752

Muench, A. A., Alves, J., Lada, Ch. J., \& Lada, E. A. 2001, ApJ, 558, 51

Natta, A., \& Testi, L. 2001, A\&A, 376, 22

Natta, A., Testi, L., Comerón, F., et al. 2002, A\&A, 393, 597

O’Dell, M. A., Hilditch, R. W., Collier Cameron, A., \& Bell, S. A. 1997, MNRAS, 284, 874

Oliveira, J. M., Jeffries, R. D., Kenyon, M. J., Thompson, S. A., \& Naylor, T. 2002, A\&A, 382, 22

Oliveira, J. M., Jeffries, R. D., \& van Loon, J. T. 2003, MNRAS, 347, 1327

Patten, B. M., \& Simon, Th. 1996, ApJS, 106, 489

Pickett, B. K., Durisen, R. H., Cassen, P., \& Mejia, A. C. 2000, ApJ, 540, 95

Pinfield, D. J., Hodgkin, S. T., Jameson, R. F., et al. 2000, MNRAS, 313,347

Rebull, L. M., Wolff, S. C., Strom, S. E., \& Makidon, R. B. 2002, AJ, 124,546

Reipurth, B., Bally, J., Graham, J. A., Lane, A. P., \& Zealey, W. J. 1986, A\&A, 164, 51

Reipurth, B., \& Clarke, C. 2001, AJ, 122, 432

Riffeser, A., Fliri, J., Gössl, C. A., et al. 2001, A\&A, 379, 362

Roberts D. H., Lehar, J., \& Dreher, J. W. 1987, AJ, 93, 968

Robin, A., \& Crézé, M. 1986, A\&A, 157, 71

Scargle, J. D. 1982, ApJ, 263, 835

Stassun, K. G., Mathieu, R. D., Mazeh, T., \& Vrba, F. J. 1999, AJ, 117,2941

Stassun, K. G., Mathieu, R. D., Vrba, F. J., Mazeh, T., \& Henden, A. 2001, AJ, 121, 1003

Stetson, P. B. 1987, PASP, 99, 191

Terndrup, D. M., Krishnamurthi, A., Pinsonneault, M. H., \& Stauffer, J. R. 1999, AJ, 118, 1814

Testi, L., Natta, A., Oliva, E., et al. 2002, ApJ, 571, 155

Tinney, C. G., \& Tolley, A. J. 1999, MNRAS, 304, 119

White, R. J., \& Basri, G. 2003, ApJ, 582, 1109

Wolk, S. J. 1996, Ph.D. Thesis, State Univ. New York at Stony Brook

Zapatero Osorio, M. R., Rebolo, R., \& Martín, E. L. 1997, A\&A, 317, 164

Zapatero Osorio, M. R., Rebolo, R., Martín, E. L., et al. 1999, A\&AS, 134, 537

Zapatero Osorio, M. R., Béjar, V. J. S., Martín, E. L., et al. 2000, Science, 290, 103

Zapatero Osorio, M. R., Béjar, V. J. S., Pavlenko, Y., et al. 2002, A\&A, 384,937 